Chapter 19

\title{
Dynamic Portfolio Choice and Risk Aversion
}

\section{Costis Skiadas}

Kellogg School of Management, Department of Finance, Northwestern University, 2001 Sheridan Road, Evanston, IL 60208, USA

E-mail: c-skiadas@kellogg.northwestern.edu

\begin{abstract}
This chapter presents a theory of optimal lifetime consumption-portfolio choice in a continuous information setting, with emphasis on the modeling of risk aversion through generalized recursive utility. A novel contribution is a decision theoretic development of the notions of source-dependent first- or second-order risk aversion. Backward stochastic differential equations (BSDEs) are explained heuristically as continuous-information versions of backward recursions on an information tree, and are used to formulate utility functions as well as optimality conditions. The role of scale invariance and quadratic BSDEs in obtaining tractable solutions is explained. A final section outlines extensions, including optimality conditions under trading constraints, and tractable formulations with nontradeable income.
\end{abstract}

\section{Introduction}

This chapter analyzes the optimal consumption-portfolio choice of a riskaverse agent, with emphasis on the modeling of risk aversion given a stochastic investment opportunity set. The main part of the analysis is based on Schroder and Skiadas (2003). A novel contribution is a decision theoretic development of the notions of source-dependent first- or second-order risk aversion that are implicit in the utility representations of Schroder and Skiadas (2003). These ideas unify, at least in the context of continuous information, standard notions of risk aversion with some models of ambiguity aversion or robustness that have recently received considerable attention in the literature. The dynamic portfolio methodology presented should, however, also be of interest to readers only concerned with conventional source-independent risk aversion in a dynamic setting.

Following Merton's $(1969,1971)$ seminal work, most papers on dynamic portfolio choice assume that the investor maximizes time-additive expected 
utility, that we refer to as "additive utility" for the purposes of this discussion. The limitations of additive utility in modeling risk aversion are, however, well recognized by now (see, for example, Epstein, 1992). We will argue that any two additive utilities that imply identical preferences over deterministic plans must be ordinally equivalent, and therefore equally risk-averse. In this chapter, we consider utility functions for which risk aversion can be adjusted without changing the utility value of deterministic plans. The stochastic setting is one in which information is revealed continuously by a finite set of Brownian motions. Utility is defined over consumption plans in terms of a single nondurable good, with an exogenous planning horizon and endowment. Markets can be incomplete, but they are sufficiently complete so that the investor's endowed income stream is tradeable, and there are no other trading constraints or transaction costs. The last section outlines extensions dealing with trading constraints and nontradeable income, and points to further extensions in the literature relaxing various combinations of the above assumptions (typically at the cost of other restrictions).

In its simplest form, the utility function we will adopt is the Duffie and Epstein (1992) continuous-information limit of the recursive utility of Kreps and Porteus (1978), which includes the widely used homothetic recursive utility specification of Epstein and Zin (1989) (a special case of which is expected discounted power or logarithmic utility ${ }^{1}$ ). In the Kreps-Porteus formulation, current utility is computed as a function of current consumption and a von Neumann-Morgenstern (1944) certainty equivalent of the continuation utility. Given sufficient smoothness, the classic analysis of small risks of Arrow $(1965,1970)$ and Pratt $(1964)$ implies that the certainty equivalent can be replaced by a quadratic criterion in the continuous-information limit, which is the reason why some elements of the original single-period mean-variance portfolio analysis of Markowitz (1952) survive in a continuous-information setting with Duffie-Epstein utility. Assuming constant relative risk aversion, the optimal portfolio is a weighted sum of an instantaneously mean-variance efficient portfolio and a hedging portfolio that accounts for the stochastic nature of the investment opportunity set (and vanishes in the case of i.i.d. instantaneous returns).

An extension of Duffie-Epstein utility we will consider allows risk aversion to depend on the source of risk. For example, investors have been documented to show a preference toward investing in the familiar: domestic stocks, firms whose products are familiar, local firms, one's employer's stock. ${ }^{2}$ The wellknown experimental findings of Ellsberg (1961), and a large literature following it, show that subjects prefer to bet on risk sources to which probabilities can

\footnotetext{
${ }^{1}$ Under some regularity, a homothetic additive utility is necessarily the additive special case of EpsteinZin utility. On the other hand, Epstein-Zin utility is only a parametric special case of the much broader class of homothetic Duffie-Epstein utilities.

${ }^{2}$ Daniel et al. (2002) survey such psychological biases in asset markets.
} 
be more unambiguously assigned, a phenomenon known as ambiguity aversion. ${ }^{3}$ One can think of risk as reflecting not only the risk that is conditional on the assumed model of the risk source, but also uncertainty about the model's validity, which is itself too difficult to model. Since model risk can vary with the source of risk, it is useful to consider source-dependent risk aversion. With this motivation, we will extend the Kreps-Porteus recursion by letting the certainty equivalent be a function of the entire vector of continuation utilities attributable to each Brownian motion separately. The locally-quadratic analysis under Duffie-Epstein utility extends to this case, but with a different coefficient of risk aversion assigned to each source of risk.

As shown in Skiadas (2003), Duffie-Epstein utility includes the "robust" specifications of Anderson et al. (2000), Hansen et al. (2001) and Maenhout (1999). Similarly, the criterion of Uppal and Wang (2003) is equivalent to a special form of recursive utility with source-dependent risk aversion (included in the "quasi-quadratic proportional aggregator" specification of Schroder and Skiadas, 2003). The multiple-prior expressions of these authors suggest a robustness interpretation of risk aversion. Conversely, their robustness interpretation of multiple-prior formulations can be thought of as risk aversion in the context of recursive utility. To avoid this semantic redundancy, in this chapter we define formally only risk aversion, and we think of robustness or ambiguity aversion as an informal consideration in selecting the degree of risk aversion toward a given source of risk.

Another way in which the Duffie-Epstein representation will be extended relates to the distinction between first- and second-order risk aversion made in a static setting by Segal and Spivak (1990). The Arrow-Pratt analysis, and by extension the Duffie-Epstein limit of Kreps-Porteus utility, relies on the smoothness of the von Neumann-Morgenstern certainty equivalent, an assumption for which there is no compelling decision-theoretic justification. Smooth expected utility implies local risk-neutrality, meaning that an investor should be willing to undertake any actuarially favorable investment in sufficiently small scale, and should be unwilling to perfectly insure a sufficiently small risk at actuarially unfavorable terms. We will consider a sourcedependent extension of Kreps-Porteus utility with nonsmooth certainty equivalent for which these conclusions are invalidated, and we will derive corresponding optimal trading strategy expressions that highlight the relationship between first-order risk aversion and portfolio holdings.

Motivated by the notion of ambiguity aversion, Epstein and Schneider (2003) formulated a multiple prior utility, whose continuous-information limit was studied by Chen and Epstein (2002). Consistent with the view of ambiguity aversion as being a form of risk aversion, the Chen-Epstein " $\kappa$-ignorance" formulation is mathematically equivalent to a case of the above mentioned extension of Duffie-Epstein utility with source-dependent first-order risk aversion.

\footnotetext{
${ }^{3}$ The view of ambiguity aversion as a form of risk aversion is further supported by the arguments of Klibanoff et al. (2002).
} 
Lazrak and Quenez (2003) analyzed the properties of a utility that is defined as a solution to a general backward stochastic differential equation (BSDE), and includes the Chen-Epstein formulation. Lazrak and Quenez made the important observation that comparative risk aversion can depend on the "direction" of risk. Complementing the Lazrak-Quenez analysis, this chapter provides a heuristic decision-theoretic foundation of their proposed utility form, that we will refer to simply as "recursive utility." The more specific models of risk aversion discussed above correspond to special functional forms of recursive utility.

Following the development of Schroder and Skiadas (2003), optimality conditions will first be derived for general concave recursive utilities, as a system of forward-backward stochastic differential equations (FBSDEs). The forward component of the system is the wealth process, which follows the investor's budget equation, and the backward components are the utility and shadowprice-of-wealth processes. The FBSDE system uncouples if the problem is scale-invariant (with respect to wealth). Combining scale-invariance with the various types of risk aversion discussed above, we will be able to formulate some interesting optimal trading strategy expressions, in terms of the solution to a single BSDE. Moreover, we will give some examples of preferences and stochastic investment opportunity sets for which the BSDE of the optimality conditions takes a quadratic form whose solution can be reduced to a tractable ODE system. A parallel theory based on translation-invariant recursive utility (which generalizes expected discounted exponential utility) can be found in Schroder and Skiadas (2005a), and is briefly discussed in the final section.

Merton approached the dynamic optimal portfolio selection problem using the Hamilton-Jacobi-Bellman equation of optimal control theory, modern expositions of which are given by Fleming and Soner (1993) and Yong and Zhou (1999). Examples of solutions with Epstein-Zin utility using this method are Giovannini and Weil (1989), Svensson (1989), Obstfeld (1994), Zariphopoulou and Tiu (2002), and Chacko and Viceira (2005). Cox and Huang (1989) and Karatzas et al. (1987) rederived the Merton solution by using the state-price density property of marginal utilities at the optimum, in a way that relied on utility additivity. This "utility gradient approach" was generalized to include recursive utilities in Duffie and Skiadas (1994), Schroder and Skiadas (1999), El Karoui et al. (2001), and Schroder and Skiadas (2003, 2005a, 2005b), and is the method adopted in this chapter. [An alternative dynamic programming derivation of the scale-invariant solutions is outlined in Schroder and Skiadas (2003).] While some further leads to the literature will be given in the final section, this chapter is not intended as a literature survey, and no attempt has been made to be comprehensive. Monographs or collected papers on dynamic portfolio choice include Merton (1990), Korn (1997), Sethi (1997), Karatzas and Shreve (1998), Gollier (2001), and Campbell and Viceira (2002). An overview of the econometrics of portfolio choice is given by Brandt (forthcoming).

The mathematical background for this chapter is outlined in the appendices of Duffie (2001), and is covered in detail by Karatzas and Shreve (1988). Less widely known are the more recent mathematical tools of BSDEs and FBSDEs, 
a general perspective on which can be found in the expositions of El Karoui et al. (1997) and Ma and Yong (1999).

The remainder of this chapter is organized in five sections. Section 2 sets up the problem and characterizes the optimum with minimal restrictions on preferences over consumption plans. Section 3 develops recursive utility, and the associated optimality conditions. Section 4 motivates some more specialized recursive utility forms, representing the various types of risk aversion introduced above. Section 5 formulates optimality conditions for these special recursive utility forms, assuming utility homotheticity. Section 6 concludes with an outline of several extensions.

\section{Optimality and state pricing}

This section introduces the stochastic setting, the investor problem, and the basic optimality verification argument in terms of the state price density property of a utility supergradient density. The essential tool of linear BSDEs is introduced in the context of state pricing. The section imposes only minimal preferences restrictions, and concludes with a discussion of the inadequacy of additive utility as a representation of risk aversion. The discussion of recursive utility begins with Section 3 .

\subsection{Dynamic investment opportunity set}

Uncertainty is represented by the probability space $(\Omega, \mathcal{F}, P)$, on which is defined a $d$-dimensional Brownian motion $B=\left(B^{1}, \ldots, B^{d}\right)^{\prime}$ over a finite time-horizon $[0, T]$. As with every vector in this chapter, $B$ is a column vector, and the prime denotes transposition. Information is represented by the (augmented) filtration $\left\{\mathcal{F}_{t}: t \in[0, T]\right\}$ generated by the Brownian motion $B$. Intuitively, we think of an information tree whose time- $t$ nodes or spots correspond to the possible paths of $B$ up to time $t$. A time-t spot is therefore a continuous function of the form $\omega^{t}:[0, t] \rightarrow \mathbb{R}^{d}$. Conditional expectation given time- $t$ information, $\mathcal{F}_{t}$, is denoted $E_{t}$. Similarly, covariance (variance) given $\mathcal{F}_{t}$ is denoted $\operatorname{cov}_{t}\left(\operatorname{var}_{t}\right)$. We assume that $\mathcal{F}=\mathcal{F}_{T}$, and therefore $E_{T}[x]=x$ for every random variable $x$.

A process in this chapter is by definition a stochastic process that is progressively measurable with respect to $\left\{\mathcal{F}_{t}\right\}$. For any process $x$, we think of the time- $t$ value $x_{t}$ (alternatively denoted $x(t)$ ) as a function of the realized spot $\omega^{t}$. In heuristic explanations (that ignore issues regarding null sets) we will write $x\left[\omega^{t}\right]$ to express this dependence. Given any subset $S$ of some Euclidean space, we let $\mathcal{L}(S)$ denote the set of processes of the form $x: \Omega \times[0, T] \rightarrow S$. For any integer $p$, typically $p=1$ or 2 , we define the set $\mathcal{L}_{p}(S)$ of all $x \in \mathcal{L}(S)$ such that $\int_{0}^{T}\left|x_{t}\right|^{p} \mathrm{~d} t<\infty$ with probability one (where $|\cdot|$ denotes Euclidean norm).

We consider a financial market allowing instantaneous default-free borrowing and lending at a continuously-compounded rate given by the process $r$. 
A dollar can be invested from time $t$ to time $t+\mathrm{d} t$ earning interest $r_{t} \mathrm{~d} t$, which is risk-free in the sense that $\operatorname{Var}_{t}\left[r_{t} \mathrm{~d} t\right]=0$, but whose value depends on time- $t$ information. For expositional simplicity, $r$ is assumed bounded (although this assumption is violated in some later applications). The rest of the market consists of trading in $m$ risky assets, whose cumulative excess returns are represented by the $m$-dimensional process $R=\left(R^{1}, \ldots, R^{m}\right)^{\prime}$. A dollar invested at time $t$ in risky asset $i$ is worth $1+r_{t} \mathrm{~d} t+\mathrm{d} R_{t}^{i}$ at time $t+\mathrm{d} t$.

We assume that $R$ is an Itô process with dynamics

$$
\mathrm{d} R_{t}=\mu_{t}^{R} \mathrm{~d} t+\sigma_{t}^{R \prime} \mathrm{d} B_{t}, \quad \mu^{R} \in \mathcal{L}_{1}\left(\mathbb{R}^{m}\right), \sigma^{R} \in \mathcal{L}_{2}\left(\mathbb{R}^{d \times m}\right) .
$$

There is, therefore, one column of $\sigma^{R}$ for every risky asset, and one row for every component of the Brownian motion $B$. The investment opportunity set is defined by the triple $\left(r, \mu^{R}, \sigma^{R}\right)$, whose value can vary from spot to spot. We think of (1) as an instantaneous linear factor model, where

$$
\begin{aligned}
& \mu_{j}^{R}(t) \mathrm{d} t=E_{t}\left[\mathrm{~d} R_{t}^{j}\right] \quad \text { and } \sigma_{i j}^{R}(t) \mathrm{d} t=\operatorname{cov}_{t}\left[\mathrm{~d} B_{t}^{i}, \mathrm{~d} R_{t}^{j}\right], \\
& i=1, \ldots, d, j=1, \ldots, m .
\end{aligned}
$$

Since $E_{t}\left[\mathrm{~d} B_{t}\right]=0$ and $E_{t}\left[\mathrm{~d} B_{t} \mathrm{~d} B_{t}^{\prime}\right]=I \mathrm{~d} t$ (where $I$ is an identity matrix) the conditional variance-covariance matrix of $\mathrm{d} R_{t}$ is

$$
E_{t}\left[\left(\mathrm{~d} R_{t}-E_{t}\left[\mathrm{~d} R_{t}\right]\right)\left(\mathrm{d} R_{t}-E_{t}\left[\mathrm{~d} R_{t}\right]\right)^{\prime}\right]=\sigma_{t}^{R \prime} \sigma_{t}^{R} \mathrm{~d} t .
$$

A time-t allocation is an $\mathcal{F}_{t}$-measurable random vector $\psi_{t}=\left(\psi_{t}^{1}, \ldots, \psi_{t}^{m}\right)^{\prime}$, where $\psi_{t}^{i}$ represents the proportion of wealth invested at time $t$ in risky asset $i$, with the remaining nonconsumed wealth invested risk-free. Negative proportions indicate short positions. The choice of a time- $t$ allocation can depend on time- $t$ information, and therefore we think of $\psi_{t}$ as a function of the realized time- $t$ spot. A dollar invested at time $t$ according to allocation $\psi_{t}$ is worth

$$
1+r_{t} \mathrm{~d} t+\psi_{t}^{\prime} \mathrm{d} R_{t}=1+\left(r_{t}+\psi_{t}^{\prime} \mu_{t}^{R}\right) \mathrm{d} t+\left(\sigma_{t}^{R} \psi_{t}\right)^{\prime} \mathrm{d} B_{t}
$$

at time $t+\mathrm{d} t$. The vector $\sigma_{t}^{R} \psi_{t}$ represents the risk profile of the allocation $\psi_{t}$, since it specifies the loadings of the instantaneous excess return $\psi_{t}^{\prime} \mathrm{d} R_{t}$ on the instantaneous factors $\mathrm{d} B_{t}$.

If the column span of $\sigma^{R}$ is $\mathbb{R}^{d}$ at all times then the market is complete, in the sense that every risk profile is feasible through some allocation at all times. We do not assume that the market is complete, allowing the rank of $\sigma^{R}$ to be less than $d$. While the market can be effectively complete even if $\sigma^{R}$ drops rank, we will consider applications in which market incompleteness is a binding constraint. We will not allow, however, the rank of $\sigma^{R}$ to vary from spot to spot, and we assume that at no spot of the information tree are any of the assets redundant over an infinitesimal time interval. This is the economic content of the following condition, assumed throughout:

Asset nonredundancy. The columns of $\sigma^{R}$, corresponding to the $m$ risky assets, are everywhere linearly independent, and therefore $m \leqslant d$. 
As a consequence of this assumption, the $m \times m$ instantaneous variancecovariance rate matrix $\sigma^{R^{\prime}} \sigma^{R}$ is everywhere invertible. If $\sigma_{t}$ is a risk profile attainable through the allocation $\psi_{t}$, meaning that $\sigma_{t}^{R} \psi_{t}=\sigma_{t}$, then $\psi_{t}$ is the unique allocation with this property, and is given by

$$
\psi_{t}=\left(\sigma_{t}^{R \prime} \sigma_{t}^{R}\right)^{-1} \sigma_{t}^{R \prime} \sigma_{t} .
$$

The traditional portfolio analysis of Markowitz (1952) can be applied conditionally spot-by-spot on the information tree. Selecting an allocation $\psi_{t}$ results in an instantaneous excess return with conditional mean and variance

$$
E_{t}\left[\psi_{t}^{\prime} \mathrm{d} R_{t}\right]=\psi_{t}^{\prime} \mu_{t}^{R} \mathrm{~d} t \quad \text { and } \quad \operatorname{var}_{t}\left[\psi_{t}^{\prime} \mathrm{d} R_{t}\right]=\psi_{t}^{\prime} \sigma_{t}^{R \prime} \sigma_{t}^{R} \psi_{t} \mathrm{~d} t .
$$

Let $\mu_{t}$ be any $\mathcal{F}_{t}$-measurable random variable. Minimizing $\operatorname{var}_{t}\left[\psi_{t}^{\prime} \mathrm{d} R_{t}\right]$ subject to the constraint $E_{t}\left[\psi_{t}^{\prime} \mathrm{d} R_{t}\right]=\mu_{t} \mathrm{~d} t$ results in an allocation of the form

$$
\psi_{t}=k_{t}\left(\sigma_{t}^{R \prime} \sigma_{t}^{R}\right)^{-1} \mu_{t}^{R},
$$

for some $\mathcal{F}_{t}$-measurable random variable $k_{t}$ that depends on the choice of $\mu_{t}$. We call an allocation of this form instantaneously minimum-variance efficient. The corresponding squared conditional instantaneous Sharpe ratio is maximized, and is given by

$$
\frac{E_{t}\left[\psi_{t}^{\prime} \mathrm{d} R_{t}\right]^{2}}{\operatorname{var}_{t}\left[\psi_{t}^{\prime} \mathrm{d} R_{t}\right]}=\mu_{t}^{R \prime}\left(\sigma_{t}^{R \prime} \sigma_{t}^{R}\right)^{-1} \mu_{t}^{R} \mathrm{~d} t
$$

\subsection{Strategies, utility, and optimality}

An optimal investment strategy is one that finances a consumption plan for which there exists no other consumption plan that is both more desirable and feasible. In this subsection we formalize this notion, while placing minimal restrictions on investor preferences.

We let $\mathcal{H}$ denote the Hilbert space of every $x \in \mathcal{L}(\mathbb{R})$ such that $E\left[\int_{0}^{T} x_{t}^{2} \mathrm{~d} t+\right.$ $\left.x_{T}^{2}\right]<\infty$, with the inner product

$$
(x \mid y)=E\left[\int_{0}^{T} x_{t} y_{t} \mathrm{~d} t+x_{T} y_{T}\right], \quad x, y \in \mathcal{H} .
$$

The set of strictly positive $e^{4}$ elements of $\mathcal{H}$ is $\mathcal{H}_{++}=\mathcal{H} \cap \mathcal{L}\left(\mathbb{R}_{++}\right)$. The element of $\mathcal{H}$ that is identically equal to one is denoted $\mathbf{1}$.

We postulate a convex cone $\mathcal{C} \subseteq \mathcal{H}_{++}$of consumption plans such that $\mathbf{1} \in \mathcal{C}$, and for any $x \in \mathcal{H}$ and $y, z \in \mathcal{C}, y \leqslant x \leqslant z$ implies $x \in \mathcal{C}$. For any $c \in \mathcal{C}$ and

\footnotetext{
${ }^{4}$ More precisely, any two processes $x, y$ such that $(x-y \mid x-y)=0$ are identified as elements of $\mathcal{H}$. A strictly positive element of $\mathcal{H}$ is one that can be identified in this way with a process in $\mathcal{L}\left(\mathbb{R}_{++}\right)$.
} 
time $t<T$, we interpret $c_{t}$ as the time- $t$ consumption rate, while $c_{T}$ represents a terminal lump-sum consumption or bequest. In a typical application, $\mathcal{C}$ is specified by some integrability restriction required for a utility function to be well defined. The strict positivity of consumption plans reflects our implicit assumption that a consumption nonnegativity constraint is nonbinding. In later sections, the positivity of optimal consumption will be enforced by assuming infinite marginal utility at zero.

We consider an investor with initial wealth $w_{0}>0$ and no subsequent income. (This includes the case of an endowed income stream as long as it can be traded.) A consumption strategy is any process $\rho \in \mathcal{L}_{1}\left(\mathbb{R}_{++}\right)$such that $\rho_{T}=1$. For $t<T$, we interpret $\rho_{t}$ as the investor's consumption rate as a proportion of time- $t$ wealth, while the convention $\rho_{T}=1$ is used below to express the assumption that final wealth equals terminal consumption. A trading strategy is any process $\psi \in \mathcal{L}\left(\mathbb{R}^{m}\right)$ such that $\psi^{\prime} \mu^{R} \in \mathcal{L}_{1}(\mathbb{R})$ and $\sigma^{R} \psi \in \mathcal{L}_{2}\left(\mathbb{R}^{d}\right)$, with $\psi_{t}$ representing a time-t allocation. A strategy is a pair $(\rho, \psi)$ of a consumption strategy and a trading strategy.

The wealth process $W$ generated by a strategy $(\rho, \psi)$ is defined through the budget equation

$$
\frac{\mathrm{d} W_{t}}{W_{t}}=\left(r_{t}-\rho_{t}\right) \mathrm{d} t+\psi_{t}^{\prime} \mathrm{d} R_{t}, \quad W_{0}=w_{0} .
$$

The consumption plan $c$ is financed by the strategy $(\rho, \psi)$ if $c=\rho W$, meaning that $c_{t}=\rho_{t} W_{t}$ for every time $t$ (and therefore $c_{T}=W_{T}$ ). A consumption plan is feasible if it can be financed by some strategy.

The investor's problem is to select a feasible consumption plan that is optimal. To define optimality, we introduce utility functions. We say that the investor prefers plan $b$ to plan $a$ at spot $\omega^{t}$ if, conditionally on the realization of $\omega^{t}$, an agent with plan $a$ as the status quo would switch to plan $b$ if presented with the opportunity to do so at no cost. The investor is indifferent between two plans if neither plan is preferred to the other.

We are going to measure utility concretely by taking as a unit the consumption plan 1. We assume throughout that the investor prefers more consumption to less, and therefore, given any scalars $\alpha, \beta$ such that $\beta>\alpha>0$, the agent prefers $\beta \mathbf{1}$ to $\alpha \mathbf{1}$ at every spot. We further assume that, given any consumption plan $c$ and spot $\omega^{t}$, there exists a (necessarily unique) scalar $\alpha$ such that, conditionally on the realization of spot $\omega^{t}$, the agent is indifferent between plans $c$ and $\alpha \mathbf{1}$. We call this value of $\alpha$ the spot- $\omega^{t}$ cardinal utility of $c$, and denote it $U(c)\left[\omega^{t}\right]$. Specifying a value at every spot of the information tree defines the cardinal utility process $U(c)$ of plan $c$. We note that, by definition, $U_{T}(c)=c_{T}$.

Another preference assumption we adopt is that if the investor is indifferent between $a$ and $a^{\prime}$ and between $b$ and $b^{\prime}$, then the investor prefers $b$ to $a$ if and only if the investor prefers $b^{\prime}$ to $a^{\prime}$. Applying this condition with $a^{\prime}=U(a)\left[\omega^{t}\right] \mathbf{1}$ and $b^{\prime}=U(b)\left[\omega^{t}\right] \mathbf{1}$, we conclude that, conditionally on the realization of spot $\omega^{t}$, the investor prefers plan $b$ to plan $a$ if and only if $U(b)\left[\omega^{t}\right]>U(a)\left[\omega^{t}\right]$. 
The investor's objective at spot $\omega^{t}$ is therefore to select the feasible consumption plan $c$ of maximum spot- $\omega^{t}$ utility $U(c)\left[\omega^{t}\right]$.

Utility maximization at every spot can be an inconsistent objective, since the investor may have an incentive to deviate at some spot from a strategy selected at an earlier spot. We exclude this possibility by assuming the following key condition throughout.

Dynamic consistency. Suppose two consumption plans $a$ and $b$ are equal up to a stopping time $\tau$, and $P\left[U_{\tau}(b) \geqslant U_{\tau}(a)\right]=1$. Then $U_{0}(b) \geqslant U_{0}(a)$, with the inequality being strict if $P\left[U_{\tau}(b)>U_{\tau}(a)\right]>0$.

Suppose time-zero utility is maximized by the strategy $(\rho, \psi)$, which finances the consumption plan $c$, and generates the wealth process $W$. Then there cannot exist a stopping time $\tau$ and trading strategy $(\tilde{\rho}, \tilde{\psi})$, that finances consumption plan $\tilde{c}$ and generates a wealth process $\tilde{W}$, such that $W_{\tau}=\tilde{W}_{\tau}$, $P\left[U_{\tau}(\tilde{c}) \geqslant U_{\tau}(c)\right]=1$, and $P\left[U_{\tau}(\tilde{c})>U_{\tau}(c)\right]>0$. Otherwise, by dynamic consistency, the strategy that starts as $(\rho, \psi)$ and switches to $(\tilde{\rho}, \tilde{\psi})$ at time $\tau$ would result in higher time-zero utility than $U_{0}(c)$, contradicting the time-zero optimality of strategy $(\rho, \psi)$.

Dynamic consistency justifies the following definition of optimality in terms of the single time-zero utility function $U_{0}: \mathcal{C} \rightarrow \mathbb{R}$.

Definition 1. The consumption plan $c$ is optimal if it is feasible and there exists no feasible consumption plan $\tilde{c}$ such that $U_{0}(\tilde{c})>U_{0}(c)$. A strategy $(\rho, \psi)$ is optimal if it finances an optimal consumption plan. Finally, a consumption or trading strategy is optimal if it is part of an optimal strategy.

A function $\tilde{U}_{0}: \mathcal{C} \rightarrow \mathbb{R}$ is ordinally equivalent to $U_{0}: \mathcal{C} \rightarrow \mathbb{R}$ if $\tilde{U}_{0}=f \circ U_{0}$ for some strictly increasing function $f: \mathbb{R} \rightarrow \mathbb{R}$. We call such a function $\tilde{U}_{0}$ an ordinal utility representation of the investor's time-zero preferences. A property of $U_{0}$ is ordinal if it is also true of any utility that is ordinally equivalent to $U_{0}$. Optimality of a given consumption plan relative to $U_{0}$ is an example of an ordinal property of $U_{0}$. (Extending the notion of ordinal equivalence to utility at every spot, we note that dynamic consistency is an ordinal property of the entire utility process $U$.)

We henceforth take as given the time-zero utility function $U_{0}: \mathcal{C} \rightarrow \mathbb{R}$, which can be either ordinal or cardinal, the distinction made only where relevant. The following two properties are assumed throughout the chapter:

Monotonicity. For any $c, c+x \in \mathcal{C}, 0 \neq x \geqslant 0$ implies $U_{0}(c+x)>U_{0}(c)$.

Concavity. For all $c^{0}, c^{1} \in \mathcal{C}, \alpha \in(0,1)$ implies $U_{0}\left(\alpha c^{1}+(1-\alpha) c^{0}\right) \geqslant$ $\alpha U_{0}\left(c^{1}\right)+(1-\alpha) U_{0}\left(c^{0}\right)$. 
Monotonicity is an ordinal property, while concavity is not. For cardinal utility, concavity can be thought of as an expression of a preference for consumption smoothing. Later we will introduce the important class of scale-invariant problems in which $U_{0}$ is assumed to have the additional ordinal property of homotheticity. A cardinal utility is homothetic if and only if it is homogeneous of degree one, in which case concavity is equivalent to the ordinal property of quasiconcavity.

Let $(\rho, \psi)$ be a candidate optimal strategy that generates the wealth process $W$ and finances the consumption plan $c=\rho W$. We will verify the optimality of $c$ by constructing a utility supergradient density at $c$ that is also a state price density at $c$. These notions are defined below.

Definition 2. (a) A process $\pi \in \mathcal{H}$ is a state price density at $c$ if $(\pi \mid x) \leqslant 0$ for any $x \in \mathcal{H}$ such that $c+x$ is a feasible consumption plan.

(b) A process $\pi \in \mathcal{H}$ is a supergradient density of $U_{0}$ at $c$ if $U_{0}(c+x) \leqslant$ $U_{0}(c)+(\pi \mid x)$ for every $x \in \mathcal{H}$ such that $c+x \in \mathcal{C}$.

Interpreting $(\pi \mid x)$ as a present value of $x$, the state-price density property states that there is no feasible incremental consumption plan relative to $c$ that has positive present value. A supergradient density can be thought of as a generalized notion of marginal utility. Since $U_{0}$ is assumed (strictly) increasing and concave, any supergradient density of $U_{0}$ is necessarily strictly positive. Given a reference plan, the state-price density property depends on the market opportunities and not on preferences, while the supergradient density property depends on preferences and not on the market opportunities.

The following observation is the basis for optimality verification in this chapter. [While we will not discuss the necessity of optimality conditions in this chapter, a simple partial converse is given in Schroder and Skiadas (2003).]

Proposition 3. Suppose $c$ is a feasible consumption plan, and $\pi \in \mathcal{H}_{++}$is a supergradient density of $U_{0}$ at $c$ that is also a state price density at $c$. Then the plan $c$ is optimal.

Proof. If $c+x \in \mathcal{C}$ is feasible, then $U_{0}(c+x) \leqslant U_{0}(c)+(\pi \mid x) \leqslant U_{0}(c)$.

\subsection{State price dynamics and linear BSDEs}

In order to apply the optimality verification argument of Proposition 3, we study below the dynamics of a state price density. In the process we introduce the mathematical tool of a linear backward stochastic differential equation (BSDE), which plays a basic role in this chapter and asset pricing theory in general.

The key to understanding the state price density dynamics is the following notion of risk pricing: 
Definition 4. A market-price-of-risk process is any process $\eta \in \mathcal{L}_{2}\left(\mathbb{R}^{d}\right)$ such that

$$
\mu^{R}=\sigma^{R \prime} \eta
$$

Recalling the linear-factor-model interpretation (1), the above equation can be thought of as (exact) factor pricing, with $\eta_{t}^{i}$ representing the time- $t$ price of instantaneous linear factor $\mathrm{d} B_{t}^{i}$. Since $\sigma^{R}$ is assumed everywhere full-rank, a market-price-of-risk process is unique if and only if $m=d$.

The existence of a market-price-of-risk process is implied by the absence of arbitrage opportunities. While a rigorous statement and proof of this claim can be found in Karatzas and Shreve (1998), it is worth recalling the essential idea. In an arbitrage-free market there cannot be an instantaneously riskless allocation with positive instantaneous excess returns; that is,

$$
\sigma_{t}^{R} \psi_{t}=0 \quad \text { implies } \quad \psi_{t}^{\prime} \mu_{t}^{R}=0 .
$$

The existence of a market price of risk process is the dual equivalent to (6). Clearly, (5) implies (6). Conversely, we define the orthogonal decomposition $\mu_{t}^{R}=\sigma_{t}^{R \prime} \eta_{t}+\varepsilon_{t}$, where $\sigma_{t}^{R} \varepsilon_{t}=0$. If (6) holds, then $\varepsilon_{t}^{\prime} \mu_{t}^{R}=0$, and therefore $\varepsilon_{t}^{\prime} \varepsilon_{t}=\varepsilon_{t}^{\prime} \mu_{t}^{R}=0$, proving that $\mu_{t}^{R}=\sigma_{t}^{R \prime} \eta_{t}$.

Suppose that the process $\pi \in \mathcal{H}_{++}$follows the dynamics

$$
\frac{\mathrm{d} \pi_{t}}{\pi_{t}}=-r_{t} \mathrm{~d} t-\eta_{t}^{\prime} \mathrm{d} B_{t}, \quad t \in[0, T],
$$

for some market-price-of-risk process $\eta$. We will argue that $\pi$ is a state-price density at any given consumption plan satisfying an integrability condition.

Consider any strategy $(\rho, \psi)$, generating the wealth process $W$, and financing the consumption plan $c=\rho W$. Letting $\Sigma=W \sigma^{R} \psi$ in the budget equation (4) and using the assumption $\mu^{R}=\sigma^{R^{\prime}} \eta$ results in

$$
\mathrm{d} W_{t}=-\left(c_{t}-r_{t} W_{t}-\eta_{t}^{\prime} \Sigma_{t}\right) \mathrm{d} t+\Sigma_{t}^{\prime} \mathrm{d} B_{t}, \quad W_{T}=c_{T} .
$$

This is a linear BSDE. The Itô process $W$ solves the BSDE if (8) is satisfied for some $\Sigma \in \mathcal{L}_{2}\left(\mathbb{R}^{d}\right)$. Given the solution $W$, the corresponding $\Sigma \in \mathcal{L}_{2}\left(\mathbb{R}^{d}\right)$ is uniquely determined (by the uniqueness of Itô representations) and therefore we can also think of a solution as being the pair $(W, \Sigma) \in \mathcal{L}_{1}(\mathbb{R}) \times \mathcal{L}_{2}\left(\mathbb{R}^{d}\right)$. Nonlinear BSDEs are introduced in the following section, where it is explained that a BSDE is essentially a backward recursion on the information tree. For the linear case, the backward recursion interpretation is suggested by a present value formula given in the lemma below. Even though the symbols have specific meanings in this context, the lemma is stated in a way that applies to a general linear BSDE.

Lemma 5. Suppose that $W$ solves BSDE (8) for some $c \in \mathcal{H}, r \in \mathcal{L}_{1}(\mathbb{R})$, and $\eta \in \mathcal{L}_{2}(\mathbb{R})$, and that $\pi \in \mathcal{H}_{++}$follows the dynamics $(7)$. 
(a) If $W \in \mathcal{L}\left(\mathbb{R}_{+}\right)$, then

$$
W_{t} \geqslant \frac{1}{\pi_{t}} E_{t}\left[\int_{t}^{T} \pi_{s} c_{s} \mathrm{~d} s+\pi_{T} c_{T}\right], \quad t \in[0, T]
$$

(b) If $E\left[\sup _{t} \pi_{t}\left|W_{t}\right|\right]<\infty$, then

$$
W_{t}=\frac{1}{\pi_{t}} E_{t}\left[\int_{t}^{T} \pi_{s} c_{s} \mathrm{~d} s+\pi_{T} c_{T}\right], \quad t \in[0, T] .
$$

Proof. Suppose $(W, \Sigma)$ satisfies (8). Integration by parts gives $\mathrm{d}(\pi W)=$ $-\pi c \mathrm{~d} t+\ldots \mathrm{d} B$. Let $\left\{\tau_{n}\right\}$ be an increasing sequence of stopping times converging to $T$ almost surely, and such that the $\ldots \mathrm{d} B$ term stopped at $\tau_{n}$ is a martingale. Integrating the last equation from $t$ to $T$, and applying the operator $E_{t}$ on both sides, we find

$$
\pi_{t} W_{t}=E_{t}\left[\int_{t}^{\tau_{n}} \pi_{s} c_{s} \mathrm{~d} s+\pi_{\tau_{n}} W_{\tau_{n}}\right] .
$$

If $W \geqslant 0$, we can take the limit as $n \rightarrow \infty$ and apply Fatou's lemma to conclude (9). If $E\left[\sup _{t} \pi_{t}\left|W_{t}\right|\right]<\infty$, then we can apply dominated convergence to conclude (10).

Remark 6. Conversely, if $W$ is given by (10), then $W$ solves BSDE (8). This can be shown by rearranging (10), and using integration by parts and a martingale representation theorem.

In our context, where $W$ is the wealth process generated by a strategy financing the consumption plan $c$, the above lemma implies the state-price-density property of $\pi$ :

Proposition 7. Suppose $\pi \in \mathcal{H}_{++}$follows the dynamics (7) for a market-priceof-risk process $\eta$. If $E\left[\sup _{t} \pi_{t} W_{t}\right]<\infty$, then $\pi$ is a state price density at $c$.

Proof. Suppose $c+x$ is a feasible consumption plan. By Lemma 5, $\pi_{0} w_{0} \geqslant$ $(\pi \mid c+x)$ and $\pi_{0} w_{0}=(\pi \mid c)$. Therefore, $(\pi \mid x) \leqslant 0$.

Remark 8. The necessity of condition (7) for an Itô process $\pi \in \mathcal{H}_{++}$to be a state price density at $c$ is shown, under some regularity assumptions, in Schroder and Skiadas (2003), where the characterization is also extended to allow for trading constraints. For example, necessity follows if $\mathcal{C}=\mathcal{H}_{++}$and $c \in \mathcal{C}$ is continuous. 
In Lemma 5, we saw that the linear term $r W+\eta^{\prime} \Sigma$ in BSDE (8) corresponds to stochastic discounting in the present value formula (10). Alternatively, the two terms can be interpreted separately, with $r W$ corresponding to temporal discounting and $\eta^{\prime} \Sigma$ corresponding to a change of measure. To see how, we define, given any $\eta \in \mathcal{L}_{2}\left(\mathbb{R}^{d}\right)$, the processes $\xi^{\eta}$ and $B^{\eta}$ by

$$
\frac{\mathrm{d} \xi_{t}^{\eta}}{\xi_{t}^{\eta}}=-\eta_{t}^{\prime} \mathrm{d} B_{t}, \quad \xi_{0}^{\eta}=1, \quad \text { and } \quad \mathrm{d} B_{t}^{\eta}=\mathrm{d} B_{t}+\eta_{t} \mathrm{~d} t, \quad B_{0}^{\eta}=0 .
$$

We recall that $\xi^{\eta}$ is a positive supermartingale, and is a martingale if and only if $E \xi_{T}^{\eta}=1$. Suppose $\eta \in \mathcal{L}_{2}\left(\mathbb{R}^{d}\right)$ is such that $\xi^{\eta}$ is a martingale. In this case an equivalent-to- $P$ probability measure $P^{\eta}$, with expectation operator $E^{\eta}$, is well defined through the change-of-measure formula $E^{\eta}[x]=E\left[\xi_{T} x\right]$ (or $\left.\mathrm{d} P^{\eta} / \mathrm{d} P=\xi_{T}\right)$. By Girsanov's theorem, $B^{\eta}$ is standard Brownian motion under $P^{\eta}$. The linear BSDE (8) can equivalently be stated as

$$
\mathrm{d} W_{t}=-\left(c_{t}-r_{t} W_{t}\right) \mathrm{d} t+\Sigma_{t}^{\prime} \mathrm{d} B_{t}^{\eta}, \quad W_{T}=c_{T} .
$$

Applying Lemma 5 and Remark 6 to this BSDE with underlying probability $P^{\eta}$, we conclude that, if $E^{\eta}\left[\sup _{t} \exp \left(-\int_{0}^{t} r_{\tau} \mathrm{d} \tau\right)\left|W_{t}\right|\right]<\infty$, then $W$ solves BSDE (8) if and only if

$$
W_{t}=E_{t}^{\eta}\left[\int_{t}^{T} e^{-\int_{t}^{s} r_{\tau} \mathrm{d} \tau} c_{s} \mathrm{~d} s+e^{-\int_{t}^{T} r_{\tau} \mathrm{d} \tau} c_{T}\right] .
$$

Equation (12) is the familiar risk-neutral-pricing version of the presentvalue formula (10), stating that financial wealth is equal to the present value of the future cash flow that this wealth finances. In a Markovian setting, such a present value can be computed (under some regularity) in terms of a corresponding PDE solution, sometimes referred to as the Feynman-Kac solution (see Duffie, 2001). The PDE form can be derived by writing $W$ as a function of time and the underlying Markov state, applying Itô's lemma, and matching terms with the linear BSDE (8). This type of construction applies more generally to BSDEs, and can be used to characterize optimal portfolios, as will be outlined for a class of scale-invariant solutions in Section 5.

\subsection{Expected time-additive utility and what is wrong with it}

Having understood the structure of state price dynamics, which is unrelated to preferences, we turn our attention to the utility side. Our objective is to specify some utility functional structure that properly captures a notion of risk aversion, and then compute the supergradient density dynamics. Combining the latter with the state price dynamics will result in optimality conditions. 
A widely used functional form of the time-zero utility function $U_{0}: \mathcal{C} \rightarrow \mathbb{R}$ is

$$
U_{0}(c)=E\left[\int_{0}^{T} e^{-\beta t} u\left(c_{t}\right) \mathrm{d} t+e^{-\beta T} v\left(c_{T}\right)\right],
$$

for some $\beta \in \mathbb{R}$ and concave increasing functions $u, v: \mathbb{R}_{++} \rightarrow \mathbb{R}$. The more concave $u$ is, the more risk-averse the utility. An advantage of this specification is that a supergradient density can be computed separately at each spot, simplifying the investor problem, at least under complete markets. For example, suppose that (13) holds with $u=v$, the derivative $u^{\prime}$ exists and maps $\mathbb{R}_{++}$onto $\mathbb{R}_{++}$, and the optimal consumption plan $c$ satisfies $u^{\prime}(c) \in \mathcal{H}_{2}$. It is straightforward to check that the process $e^{-\beta t} u^{\prime}\left(c_{t}\right)$ is a supergradient density of $U_{0}$ at $c$. If the market is complete $(m=d)$, then there exists a unique state price density $\pi$ with $\pi_{0}=1$, given by the dynamics (7) with $\eta=\sigma^{R /-1} \mu^{R}$. The optimal consumption is $c_{t}=u^{\prime-1}\left(e^{\beta t} k \pi_{t}\right)$, where the scalar $k>0$ is selected so that $(\pi \mid c)=w_{0}$. The corresponding wealth process $W$ is given by the present value formula (10). If $\mathrm{d} W / W=\ldots \mathrm{d} t+\sigma^{\prime} \mathrm{d} B$, then we have seen that the corresponding optimal trading strategy $\psi$ is given by (2). This is essentially the analysis of the Merton problem by Cox and Huang (1989) and Karatzas et al. (1987). Later in this chapter, we will see that much of the simplicity of the above argument is lost if markets are incomplete.

We argue that, despite its popularity, the time-additive utility specification (13) is fundamentally flawed as a representation of risk aversion, which is a good reason for investing some effort in studying recursive utility. Emphasizing the temporal aspect of consumption, we focus in the remainder of this section on preferences over consumption plans with fixed terminal consumption or bequest, and we therefore assume that (13) holds with $v=0$. We show below that in this case the investor's preferences over deterministic choices determine, up to ordinal equivalence, the investor's entire utility function, and in particular the investor's risk aversion. On the other hand, we will see that with recursive utility two investors can have identical preference in a deterministic environment, and yet one investor can be more risk-averse than the other.

We use the following standard uniqueness result from additive representation theory. A proof can be found in Narens (1985) or Wakker (1989).

Lemma 9. For any integer $N>1$, and each $i \in\{1,2\}$, suppose the function $F^{i}: \mathbb{R}_{++}^{N} \rightarrow \mathbb{R}$ has the additive structure $F^{i}\left(x_{1}, \ldots, x_{N}\right)=\sum_{n=1}^{N} f_{n}^{i}\left(x_{n}\right), x \in$ $\mathbb{R}_{++}^{N}$, where $f_{n}^{i}: \mathbb{R}_{++} \rightarrow \mathbb{R}, n=1, \ldots, N$. Suppose also that $F^{1}$ and $F^{2}$ are ordinally equivalent, meaning that $F^{1}(x) \geqslant F^{1}(y)$ if and only if $F^{2}(x) \geqslant F^{2}(y)$. Then there exists $\alpha \in \mathbb{R}_{++}$and $\beta \in \mathbb{R}^{N}$ such that $f_{n}^{1}=\alpha f_{n}^{2}+\beta_{n}, n=1, \ldots, N$.

Proposition 10. For each $i \in\{1,2\}$, suppose the utility function $U_{0}^{i}: \mathcal{C} \rightarrow \mathbb{R}$ takes the form $U_{0}^{i}(c)=E \int_{0}^{T} v^{i}\left(t, c_{t}\right) \mathrm{d} t$, where $v^{i}:[0, T] \times \mathbb{R}_{++} \rightarrow \mathbb{R}$ is continuous. 
Suppose also that, for any deterministic ${ }^{5}$ consumption plans $a, b \in \mathcal{C}, U_{0}^{1}(a) \geqslant$ $U_{0}^{1}(b)$ if and only if $U_{0}^{2}(a) \geqslant U_{0}^{2}(b)$. Then the utility functions $U_{0}^{1}$ and $U_{0}^{2}$ are ordinally equivalent on the entire space $\mathcal{C}$.

Proof. After replacing $U_{0}^{i}$ with $U_{0}^{i}-U_{0}^{i}(\mathbf{1})$, we can and do assume that $v^{i}(t, 1)=0$ for all $t$. Given any integer $N>1$, we define the time intervals $J^{n}=[(n-1) T / N, n T / N), n=1, \ldots, N$, partitioning [0,T). Let $D_{N}$ be the set of deterministic plans of the form $\sum_{n=1}^{N} x_{n} 1_{J^{n}}$. Since $U_{0}^{1}$ and $U_{0}^{2}$ order the elements of $D_{N}$ the same, we can apply the above lemma with $f_{n}^{i}(x)=\int_{J^{n}} v^{i}(t, x) \mathrm{d} t$ to conclude that, for some $\alpha \in \mathbb{R}_{++}$and all $n$,

$$
\int_{J^{n}} v^{1}(t, x) \mathrm{d} t=\alpha \int_{J^{n}} v^{2}(t, x) \mathrm{d} t .
$$

Repeating the above argument with $2 N$ in place of $N$ results in the same relationship, with the same constant $\alpha$, since $D_{N}$ can be embedded into $D_{2 N}$. For any $x>0$, we can therefore take a sequence of intervals $\left\{J^{n}: n=1,2, \ldots\right\}$ containing $x$, whose length converges to zero and (14) holds for all $n$. Dividing both sides of (14) by the length of $J_{n}$ and taking the limit as $n \rightarrow \infty$, we conclude that $v^{1}(t, x)=\alpha v^{2}(t, x)$.

The limitation of additive utility in capturing risk aversion is illustrated in the following variant of what seems to be a folklore example (which I learned from Duffie and Epstein, 1992). ${ }^{6}$

Example 11. Suppose that $T=100$ and $U_{0}(c)=E\left[\int_{0}^{100} v\left(t, c_{t}\right) \mathrm{d} t\right]$ for some continuous function $v:[0,100] \times \mathbb{R}_{++} \rightarrow \mathbb{R}$. The plans $a$ and $b$ are defined by

$$
\begin{aligned}
& a_{t}=1+1,000 \times 1_{\left\{t>1, B_{1}>0\right\}} \text { and } \\
& b_{t}=1+1,000 \times \sum_{n=1}^{99} 1_{\left\{1+n \geqslant t>n, B_{n}-B_{n-1}>0\right\}} .
\end{aligned}
$$

While $E a_{t}=E b_{t}$ for all $t$, one could reasonably argue that plan $b$ is less risky than plan $a$. Yet, it is straightforward to check that $U_{0}(a)=U_{0}(b)$.

\footnotetext{
${ }^{5}$ We call a process $x$ deterministic if $x_{t}$ is $\mathcal{F}_{0}$-measurable for every time $t$.

${ }^{6}$ In their introduction, Duffie and Epstein (1992) give another example of the limitation of additive utility that is based on the notion of preferences for the timing of resolution of uncertainty of Kreps and Porteus (1978). The notion was extended in Skiadas (1998) in terms of preferences over pairs of consumption plans and information streams (filtrations). Additivity relates to the nondependence of utility on the filtration argument.
} 


\section{Recursive utility}

In this section, we motivate and define recursive utility, and we derive its basic properties. By combining a computation of the utility supergradient dynamics with last section's state price dynamics, we obtain optimality conditions under recursive utility as a FBSDE system. Finally, we introduce homothetic recursive utility and its role in uncoupling the FBSDE system of the optimality conditions. Continuous-time recursive utility was first defined and analyzed by Duffie and Epstein (1992), who imposed some special structure that is useful in modeling risk aversion. Following Lazrak and Quenez (2003), we adopt a broader definition of recursive utility as a solution to a general BSDE. In the following section, we will see that the broader definition of recursive utility allows for interesting models of risk aversion that go beyond the Duffie-Epstein specification.

\subsection{Recursive utility and BSDEs}

We begin with a heuristic derivation from general principles of recursive utility. The argument should also help clarify the interpretation of a BSDE as a continuous-time representation of a backward recursion on an information tree.

We consider the cardinal dynamic utility function $U: \mathcal{C} \rightarrow \mathbb{R}$ that was informally constructed in terms of preferences in Section 2.2, and we assume that $U(c)$ is an Itô process for every $c \in \mathcal{C}$. In addition to our earlier assumptions of dynamic consistency, monotonicity, and concavity, we impose the following simplifying restriction.

Irrelevance of past or unrealized consumption. For any consumption plans $a$ and $b$, any time $t \leqslant T$, and any event $A \in \mathcal{F}_{t}$, if $a=b \operatorname{on}^{7} A \times[t, T]$, then $U(a)=U(b)$ on $A \times[t, T]$.

This assumption is not an essential aspect of a recursive utility structure, but serves as a natural benchmark in an analysis whose main focus is risk aversion. Together with dynamic consistency, it implies that, for any consumption plan $c$ and times $t<u$, the restriction of $U_{t}(c)$ on a time- $t$ event $A$ can be expressed as a function of the restriction of $c$ on $A \times[t, u]$ and the restriction of $U_{u}(c)$ on $A$. More formally, we can show ${ }^{8}$ :

\footnotetext{
${ }^{7}$ This means that the indicator of $\{(\omega, u): \omega \in A, u \in[t, T], a(\omega, u) \neq b(\omega, u)\}$ is zero as an element of $\mathcal{H}$.

${ }^{8}$ Proof. Let $D=A \cap\left\{U_{t}(a)>U_{t}(b)\right\}$, and define the stopping times $\sigma=t 1_{D}+T 1_{\Omega \backslash D}$ and $\tau=$ $u 1_{D}+T 1_{\Omega \backslash D}$. We define the plan $a^{\prime}$ (respectively $b^{\prime}$ ) to be equal to $a$ (respectively $b$ ) on $D \times[t, T]$, and some arbitrary plan $c$ outside $D \times[t, T]$. Since $a=a^{\prime}$ on $[\sigma, T]$, we have $U(a)=U\left(a^{\prime}\right)$ on $[\sigma, T]$, and therefore $U_{\tau}\left(a^{\prime}\right)=U_{\tau}(a)$ a.s. Analogously, $U_{\tau}\left(b^{\prime}\right)=U_{\tau}(b)$ a.s., and therefore $U_{\tau}\left(a^{\prime}\right)=U_{\tau}\left(b^{\prime}\right)$ a.s. Since $a^{\prime}$ and $b^{\prime}$ are equal up to the stopping time $\tau$, dynamic consistency implies $U_{0}\left(a^{\prime}\right)=U_{0}\left(b^{\prime}\right)$.
} 
Lemma 12. Given any times $t<u \leqslant T$ and event $A \in \mathcal{F}_{t}$, suppose that the consumption plans $a$ and $b$ are equal on $A \times[t, u]$ and $U_{u}(a)=U_{u}(b)$ on $A$. Then $U_{t}(a)=U_{t}(b)$ on $A$.

Proceeding heuristically, we apply the above functional relationship with the time-event $(t, A)$ corresponding to a single spot $\omega^{t}$ and $u=t+\mathrm{d} t$, where $\mathrm{d} t$ is an infinitesimal time-interval. Fixing any $c \in \mathcal{C}$, we let $U=U(c)$. Given the instantaneous factor decomposition

$$
\begin{aligned}
U_{t+\mathrm{d} t} & =m_{t}+\Sigma_{t}^{\prime} \mathrm{d} B_{t}, \quad \text { where } \\
m_{t} & =E_{t}\left[U_{t+\mathrm{d} t}\right] \quad \text { and } \quad \Sigma_{t}^{i}=\operatorname{cov}_{t}\left[U_{t+\mathrm{d} t}, \mathrm{~d} B_{t}^{i}\right], \quad i=1, \ldots, d,
\end{aligned}
$$

we obtain the functional restriction

$$
U_{t}=\Phi\left(t, c_{t}, m_{t}, \Sigma_{t}\right),
$$

for some (possibly state-dependent) function $\Phi: \Omega \times[0, T] \times \mathbb{R}_{++} \times \mathbb{R}^{1+d} \rightarrow$ $(0, \infty)$ that is adapted to the underlying information structure. Utility monotonicity and concavity heuristically imply ${ }^{9}$ that $\Phi(\omega, t, c, m, \Sigma)$ is increasing in $(c, m)$ and concave in $(c, m, \Sigma)$. Given $U_{t+d t}$, Equation (16), with $m_{t}$ and $\Sigma_{t}$ defined in (15), is used to compute $U_{t}$. Equation (16) is therefore a heuristic backward recursion on the information tree, which determines the entire utility process $U$ given the terminal value $U_{T}$.

To formulate a rigorous version of the utility recursion, we assume that the function $F$, called an (infinitesimal) aggregator, is implicitly defined, at any state $\omega$ and time $t<T$, by

$$
\mu=-F(\omega, t, c, U, \Sigma) \quad \Longleftrightarrow \quad U=\Phi(\omega, t, c, U+\mu \mathrm{d} t, \Sigma) .
$$

By monotonicity of $\Phi$ in the conditional mean argument, there is at most one value $\mu$ satisfying the right-hand side equation in (17), and therefore $F$ is uniquely determined given $\Phi$. Moreover, the monotonicity and concavity properties of $\Phi$ imply that $F(\omega, t, c, U, \Sigma)$ is increasing in $c$ and concave in $\left(c, U, \Sigma\right.$ ). (If $\Phi$ is strictly concave in $m$ it also follows ${ }^{10}$ that $F$ is decreasing in $U$. We will not need to assume this condition, although it is helpful in verifying technical regularity conditions.) We use the notation $U_{T}=F\left(T, c_{T}\right)$ to

\footnotetext{
On the other hand, $a^{\prime}$ and $b^{\prime}$ are equal up to $\sigma, U_{\sigma}\left(a^{\prime}\right)>U_{\sigma}\left(b^{\prime}\right)$ on $D$, and $U_{\sigma}\left(a^{\prime}\right)=U_{\sigma}\left(b^{\prime}\right)$ on $\Omega \backslash D$. If $P(D)>0$, then dynamic consistency would imply $U_{0}\left(a^{\prime}\right)>U_{0}\left(b^{\prime}\right)$, a contradiction. Therefore $P(D)=0$. This shows $U_{t}(a) \leqslant U_{t}(b)$ on $A$. The reverse inequality is true by symmetry.

${ }^{9}$ The idea is that the dependence of $\Phi(\omega, t, c, m, \Sigma)$ on $(c, m, \Sigma)$ is through the pair $(c, U)$, where $U=$ $m+\Sigma^{\prime} \mathrm{d} B$. One can heuristically identify $(c, U)$ with a plan that is equal to $c$ at spot $\omega^{t}$ (corresponding to $(\omega, t))$, takes the value $U$ on $[t+\mathrm{d} t, T]$ conditionally on spot $\omega^{t}$ having occurred, and it takes, say, the value one at all remaining spots. Utility monotonicity and concavity over the set of such plans translates to the corresponding properties for $\Phi(\omega, t, \cdot)$.

${ }^{10}$ To see that, make a plot of $\Phi(\omega, t, c, U+\mu \mathrm{d} t, \Sigma)$ as a function of $U$. The concave graph intersects the $45^{\circ}$ line at $U$. As $\mu$ increases, the graph moves up and the intersection with the $45^{\circ}$ line moves to the right.
} 
express the dependence of terminal utility on terminal consumption (which is the identity for cardinal utility).

Assuming the Itô decomposition $\mathrm{d} U=\mu \mathrm{d} t+\Sigma^{\prime} \mathrm{d} B$, and therefore $m=U+$ $\mu \mathrm{d} t$, recursion (16) is equivalent to the drift restriction $\mu_{t}=-F\left(t, c_{t}, U_{t}, \Sigma_{t}\right)$, resulting in the utility dynamics

$$
\mathrm{d} U_{t}=-F\left(t, c_{t}, U_{t}, \Sigma_{t}\right) \mathrm{d} t+\Sigma_{t}^{\prime} \mathrm{d} B_{t}, \quad U_{T}=F\left(T, c_{T}\right) .
$$

Equation (18) is a BSDE to be solved jointly in the (adapted) process pair $(U, \Sigma)$. The function $f(\omega, t, y, z)=F(\omega, t, c(\omega, t), y, z)$, is known as the BSDE driver. We say that the Itô process $U$ solves BSDE (18) if there exists a (necessarily unique) $\Sigma \in \mathcal{L}_{2}\left(\mathbb{R}^{d}\right)$ such that (18) is satisfied. While we have motivated BSDE (18) in terms of cardinal utility, it can also be used to characterize other ordinally equivalent utility versions, as in the following example.

Example 13 (Expected discounted utility). In the above heuristic argument, suppose

$$
\begin{aligned}
& \Phi(\omega, t, c, m, \Sigma)=u(\omega, t, c) \mathrm{d} t+m \exp (-\beta(\omega, t) \mathrm{d} t), \\
& F(\omega, t, c, U, \Sigma)=u(\omega, t, c)-\beta(\omega, t) U, \quad t<T .
\end{aligned}
$$

By Lemma 5, under a regularity assumption, the solution to BSDE (18) is

$$
U_{t}=E_{t}\left[\int_{t}^{T} \exp \left(-\int_{t}^{s} \beta_{\tau} \mathrm{d} \tau\right) u\left(s, c_{s}\right) \mathrm{d} s+\exp \left(-\int_{t}^{T} \beta_{\tau} \mathrm{d} \tau\right) F\left(T, c_{T}\right)\right] .
$$

Initial BSDE existence and uniqueness results, based on the type of Lipschitz-growth assumptions on the driver familiar from SDE theory, were first obtained by Pardoux and Peng (1990) and Duffie and Epstein (1992). An improved version of the Pardoux-Peng argument can be found in El Karoui et al. (1997). These conditions are violated in our main homothetic application to follow, which includes the widely used Epstein-Zin utility [a continuous-time version of the recursive utility parametrization used in Epstein and Zin (1989)]. Existence, uniqueness and basic properties for continuous-time Epstein-Zin utility were shown in Appendix A of Schroder and Skiadas (1999). BSDE theory has been further developed by Hamadene (1996), Lepeltier and Martin (1997, 1998, 2002), Kobylanski (2000), and others (see also El Karoui and Mazliak, 1997). Moreover, the numerical solution of BSDEs has received increasing attention, with contributions by Douglas Jr. et al. (1996), Chevance (1997), Bally and Pages (2002), Ma et al. (2002), Zhang (2004), Bouchard and Touzi (2004), Bouchard and Elie (2005), Gobet et al. (2005), Lemor et al. (2006), and others. Issues of existence, uniqueness, or numerical computation will not be further addressed in this chapter.

Given the above motivation, we now formally define the utility class used in the remainder of this chapter. We assume that utility takes values in an open 
interval $I_{U} \subseteq \mathbb{R}$, which is equal to $\mathbb{R}_{++}$for cardinal utility. Utility processes will be assumed to be members of a linear subspace $\mathcal{U} \subseteq \mathcal{L}\left(I_{U}\right)$, taken as a primitive. We assume throughout that every $U \in \mathcal{U}$ is an Itô process and satisfies $E\left[\sup _{t} U_{t}^{2}\right]<\infty$. Below we define a dynamic utility, meaning that an entire utility process $U(c)$ is assigned to a plan $c$. Later we will verify that dynamic consistency is satisfied, and is therefore sufficient to maximize timezero utility.

Definition 14. An (increasing in consumption and concave) aggregator is a progressively measurable function of the form $F: \Omega \times[0, T] \times \mathbb{R}_{++} \times I_{U} \times \mathbb{R}^{d} \rightarrow \mathbb{R}$ satisfying:

1. $F(\omega, t, c, U, \Sigma)$ is strictly increasing in $c$ and concave in $(c, U, \Sigma)$.

2. $F(\omega, T, c, U, \Sigma)$ does not depend on $(U, \Sigma)$, and is therefore denoted $F(\omega, T, c)$.

The function $U: \mathcal{C} \rightarrow I_{U}$ is recursive utility with aggregator function $F$ if, for any $c \in \mathcal{C}, U(c)$ solves BSDE (18) uniquely in $\mathcal{U}$. The aggregator $F$ is deterministic if it does not depend on the state variable. The recursive utility $U$ is state-independent if the corresponding aggregator $F$ is deterministic, and for any deterministic plan $c, U(c)$ is the unique deterministic element of $\mathcal{U}$ solving the ODE $\mathrm{d} U_{t}=-F\left(t, c_{t}, U_{t}, 0\right) \mathrm{d} t, U_{T}=F\left(T, c_{T}\right)$.

Remark 15 (Aggregator and beliefs). Suppose that $U$ is recursive utility with aggregator $F$, and the process $b \in \mathcal{L}\left(\mathbb{R}^{d}\right)$ is (for simplicity) bounded. Consider the modified aggregator

$$
F^{b}(\omega, t, c, U, \Sigma)=F(\omega, t, c, U, \Sigma)+b(\omega, t)^{\prime} \Sigma .
$$

Recalling the notation in (11), we note that

$$
\begin{aligned}
& \mathrm{d} U_{t}=-F^{b}\left(t, c_{t}, U_{t}, \Sigma_{t}\right) \mathrm{d} t+\Sigma_{t}^{\prime} \mathrm{d} B_{t}^{b} \quad \text { and } \\
& \mathrm{d} R_{t}=\left(\mu_{t}^{R}-\sigma_{t}^{R \prime} b_{t}\right) \mathrm{d} t+\sigma_{t}^{R \prime} \mathrm{d} B_{t}^{b} .
\end{aligned}
$$

Since $B^{b}$ is Brownian motion under the probability $P^{b}$ (where $\mathrm{d} P^{b} / \mathrm{d} P=\xi_{T}^{b}$ ), an investor with prior $P^{b}$ still assesses the same risk profile $\sigma^{R}$, but believes that the instantaneous expected returns are $\mu^{R}-\sigma^{R \prime} b$. A solution method to the investor's problem for $b=0$ extends to any value of $b$ after the formal substitution $\left(P, B, \mu^{R}\right) \rightarrow\left(P^{b}, B^{b}, \mu^{R}-\sigma^{R \prime} b\right)$.

\subsection{Some basic properties of recursive utility}

In this subsection we derive, under regularity assumptions, some basic properties of recursive utility. We first verify dynamic consistency, monotonicity, concavity, and the irrelevance of past or unrealized alternatives. We then discuss comparative risk aversion, and finally we compute the dynamics of a utility supergradient density. 
The following notation will be useful. For any function of the form $f: \Omega \times$ $[0, T] \times S \rightarrow \mathbb{R}$, where $S$ is a convex subset of some Euclidean space $X$, we define the superdifferential notation:

$$
\begin{gathered}
\partial f(\omega, t, s)=\left\{\delta \in X: f(\omega, t, s+h) \leqslant f(\omega, t, s)+\delta^{\prime} h\right. \\
\text { for all } h \in X \text { such that } s+h \in S\} .
\end{gathered}
$$

Given any processes $d \in \mathcal{L}(X)$ and $x \in \mathcal{L}(S)$, the notation $d \in \partial f(x)$ means that the indicator function of the set of all $(\omega, t)$ such that $d(\omega, t) \notin$ $\partial f(\omega, t, x(\omega, t))$ is the zero element of $\mathcal{H}$. Given any $d=(a, b) \in \mathcal{L}_{1}(\mathbb{R}) \times$ $\mathcal{L}_{2}\left(\mathbb{R}^{d}\right)$, we let $\mathcal{E}(d)$ or $\mathcal{E}(a, b)$ denote the stochastic exponential with dynamics

$$
\frac{\mathrm{d} \mathcal{E}_{t}(a, b)}{\mathcal{E}_{t}(a, b)}=a_{t} \mathrm{~d} t+b_{t}^{\prime} \mathrm{d} B_{t}, \quad \mathcal{E}_{0}(a, b)=1 .
$$

The key to deriving properties of recursive utility is the so-called comparison principle, stated below in terms of the (progressively measurable) driver functions $f^{i}: \Omega \times[0, T] \times I_{U} \times \mathbb{R}^{d} \rightarrow \mathbb{R}, i=0,1$.

Condition 16 (Comparison principle). For each $i \in\{0,1\}$, suppose $\left(U^{i}, \Sigma^{i}\right) \in$ $\mathcal{U} \times \mathcal{L}\left(\mathbb{R}^{d}\right)$ solves the BSDE

$$
\mathrm{d} U_{t}^{i}=-f^{i}\left(t, U_{t}^{i}, \Sigma_{t}^{i}\right) \mathrm{d} t+\Sigma_{t}^{i \prime} \mathrm{d} B, \quad t \in[0, T], U_{T}^{i} \text { given. }
$$

Given stopping times $\sigma, \tau$ such that $\sigma \leqslant \tau$ a.s., suppose also that ${ }^{11}$

$$
f^{0}\left(t, U^{1}, \Sigma^{1}\right) \leqslant f^{1}\left(t, U^{1}, \Sigma^{1}\right) \quad \text { on }[\sigma, \tau] \quad \text { and } \quad U_{\tau}^{0} \leqslant U_{\tau}^{1} \quad \text { a.s. }
$$

Then $U_{\sigma}^{0} \leqslant U_{\sigma}^{1}$ a.s. Assuming further that $P\left[U_{\tau}^{0}<U_{\tau}^{1}\right]>0$, then $P\left[U_{\sigma}^{0}<\right.$ $\left.U_{\sigma}^{1}\right]>0$.

A comparison lemma (or stochastic Gronwall-Bellman inequality in the language of Duffie and Epstein) imposes sufficient regularity restrictions for the comparison principle to hold. Various comparison lemmas are given in the BSDE literature referenced earlier. We show below an apparently new version whose applicability relies on our concavity assumption.

Lemma 17 (Comparison lemma). The comparison principle (Condition 16) holds if there exists some $d \in \mathcal{L}_{1}(\mathbb{R}) \times \mathcal{L}_{2}\left(\mathbb{R}^{d}\right)$ such that $d \in \partial f^{0}\left(U^{1}, \Sigma^{1}\right)$ a.e. and $E\left[\sup _{t} \mathcal{E}_{t}(d)^{2}\right]<\infty$.

\footnotetext{
${ }^{11}$ For processes $x, y$, we say that $x \leqslant y$ on $[\sigma, \tau]$ if the indicator function of the set of all $(\omega, t)$ such that $x(\omega, t)>y(\omega, t)$ and $\sigma(\omega) \leqslant t \leqslant \tau(\omega)$ is zero as an element of $\mathcal{H}$.
} 
Proof. Notationally suppressing the arguments $(\omega, t)$, we define the processes $y=U^{1}-U^{0} \in \mathcal{U}, z=\Sigma^{1}-\Sigma^{0}$, and $p=f^{1}\left(U^{1}, \Sigma^{1}\right)-f^{0}\left(U^{1}, \Sigma^{1}\right)$, and we note that

$$
\mathrm{d} y=-\left(f^{0}\left(U^{1}, \Sigma^{1}\right)-f^{0}\left(U^{0}, \Sigma^{0}\right)+p\right) \mathrm{d} t+z^{\prime} \mathrm{d} B .
$$

Let $d=\left(\mathrm{d}_{U}, \mathrm{~d}_{\Sigma}\right)$ be as in the lemma's statement, and define the process $q=$ $f^{0}\left(U^{1}, \Sigma^{1}\right)-f^{0}\left(U^{0}, \Sigma^{0}\right)-\left(\mathrm{d}_{U} y+\mathrm{d}_{\Sigma}^{\prime} z\right)$. Then the above dynamics for $y$ can be restated as

$$
\mathrm{d} y=-\left(\delta+\mathrm{d}_{U} y+\mathrm{d}_{\Sigma}^{\prime} z\right) \mathrm{d} t+z^{\prime} \mathrm{d} B,
$$

where $\delta=p+q$. Our assumptions imply that $\delta \geqslant 0$ on $[\sigma, \tau]$ and $y_{\tau} \geqslant 0$ a.s. Arguing as in the proof of Lemma 5, we can select a sequence of stopping times $\left\{\tau_{n}\right\}$ such that $\tau_{n} \uparrow \tau$ a.s. and

$$
\mathcal{E}_{\sigma}(d) y_{\sigma}=E_{\sigma}\left[\int_{\sigma}^{\tau_{n}} \mathcal{E}_{t}(d) \delta_{t} \mathrm{~d} t+\mathcal{E}_{\tau_{n}}(d) y_{\tau_{n}}\right] \geqslant E_{\sigma}\left[\mathcal{E}_{\tau_{n}} y_{\tau_{n}}\right] \quad \text { a.s. }
$$

We recall that, by assumption, $y \in \mathcal{U}$ implies $E\left[\sup _{t} y_{t}^{2}\right]<\infty$. Letting $n \rightarrow \infty$, and using dominated convergence, it follows that $y_{\sigma} \geqslant 0$ a.s.

Next we introduce a regularity condition that will allow us to apply the comparison lemma to derive the utility properties we are interested in. The reader who wants to skip technicalities can read "regular" as meaning "we can apply the comparison principle where we have to."

Given any aggregator $F$ and $c \in \mathcal{C}$, we use the notation $F^{c}(\omega, t, y, z)=$ $F(\omega, t, c(\omega, t), y, z)$. We call an aggregator $F$ regular if, given any $(c, U) \in$ $\mathcal{C} \times \mathcal{U}$ with $\mathrm{d} U=\ldots \mathrm{d} t+\Sigma^{\prime} \mathrm{d} B$, there exists $d \in \mathcal{L}_{1}(\mathbb{R}) \times \mathcal{L}_{2}\left(\mathbb{R}^{d}\right)$ such that $d \in \partial F^{c}(U, \Sigma)$ a.e. and $E\left[\sup _{t} \mathcal{E}_{t}(d)^{2}\right]<\infty$. For example, suppose $F$ is differentiable and $F_{U} \leqslant 0$ [which follows from the strict concavity of $\Phi$ in Equation (16)]. In this case, regularity of $F$ becomes an integrability restriction on $F_{\Sigma}$, which is satisfied if $F_{\Sigma}$ is bounded. Boundedness of $F_{\Sigma}$ is usually too strong an assumption, however, and confirming regularity is more challenging.

Proposition 18. A recursive utility with a regular aggregator is dynamically consistent, monotonically increasing, concave, and satisfies the irrelevance of past or unrealized alternatives condition.

Proof. Suppose $U$ is recursive utility with aggregator $F$, and $c^{0}, c^{1} \in \mathcal{C}$. We use the notation $U^{i}=U\left(c^{i}\right)$ and $\mathrm{d} U^{i}=\ldots \mathrm{d} t+\Sigma^{i \prime} \mathrm{d} B$. To show monotonicity, suppose $c^{1} \geqslant c^{0}$. The comparison lemma with $f^{i}=F^{c^{i}}$ implies $U^{1} \geqslant U^{0}$. To show concavity, we fix any $\alpha \in(0,1)$ and define the notation $x^{\alpha}=(1-\alpha) x^{0}+$ $\alpha x^{1}$. Notationally suppressing the arguments $(\omega, t)$, we define the process $p=$ $F\left(c^{\alpha}, U^{\alpha}, \Sigma^{\alpha}\right)-(1-\alpha) F\left(c^{0}, U^{0}, \Sigma^{0}\right)-\alpha F\left(c^{1}, U^{1}, \Sigma^{1}\right)$, and note that

$$
\mathrm{d} U^{\alpha}=-\left(F\left(c^{\alpha}, U^{\alpha}, \Sigma^{\alpha}\right)-p\right) \mathrm{d} t+\Sigma^{\alpha} \mathrm{d} B, \quad U_{T}^{\alpha}=F\left(T, c_{T}^{\alpha}\right)-p_{T} .
$$


The concavity assumption on $F$ implies that $p \geqslant 0$. Applying Lemma 17 with $f^{1}=F^{c^{\alpha}}$ and $f^{0}=F^{c^{\alpha}}-p$, we conclude that $U\left(c^{\alpha}\right) \geqslant U^{\alpha}$, confirming concavity. The remaining claims are left as an exercises in the application of Lemma 17.

A state-independent recursive utility with aggregator $F$ ranks deterministic plans in a way determined by the function $(t, c, U) \mapsto F(t, c, U, 0)$, while the dependence of $F$ on $\Sigma$ can be used to adjust risk aversion without affecting the utility of deterministic plans. The formal statement of this property is based on the following partial order of utility functions.

Comparative risk aversion. A utility function $U_{0}^{1}: \mathcal{C} \rightarrow \mathbb{R}$ is more risk-averse than a utility function $U_{0}^{2}: \mathcal{C} \rightarrow \mathbb{R}$ if

- For any deterministic plans $a, b \in \mathcal{C}$,

$$
U_{0}^{1}(a) \geqslant U_{0}^{1}(b) \quad \Longleftrightarrow \quad U_{0}^{2}(a) \geqslant U_{0}^{2}(b) .
$$

- For any $c \in \mathcal{C}$ and deterministic $\bar{c} \in \mathcal{C}$,

$$
U_{0}^{2}(\bar{c}) \geqslant U_{0}^{2}(c) \quad \Longrightarrow \quad U_{0}^{1}(\bar{c}) \geqslant U_{0}^{1}(c) .
$$

Remark 19. If $U_{0}^{1}$ and $U_{0}^{2}$ are cardinal utilities, then $U_{0}^{1}$ is more risk-averse than $U_{0}^{2}$ if and only if $U_{0}^{1}(c)=U_{0}^{2}(c)$ for every deterministic plan $c$, and $U_{0}^{1}(c) \leqslant U_{0}^{2}(c)$ for every plan $c$.

Proposition 20. Suppose that, for $i \in\{1,2\}, U^{i}$ is state-independent recursive utility with aggregator $F^{i}$, and $F^{1}$ is regular. If $F^{1}(t, c, U, 0)=F^{2}(t, c, U, 0)$ and $F^{1}(t, c, U, \Sigma) \leqslant F^{2}(t, c, U, \Sigma)$ for all $(t, c, U, \Sigma)$, then $U_{0}^{1}$ is more risk-averse than $U_{0}^{2}$.

Proof. By definition, $F^{1}(t, c, U, 0)=F^{2}(t, c, U, 0)$ implies that $U^{1}(c)=$ $U^{2}(c)$ for every deterministic plan $c$. The proof is completed using Lemma 17.

Finally, we derive a utility supergradient density expression for recursive utility, which will be key in establishing optimality conditions.

Proposition 21. Suppose $U$ is recursive utility with aggregator $F$ such that $F_{c}, F_{U} \in \mathcal{L}_{1}(\mathbb{R})$ and $F_{\Sigma} \in \mathcal{L}_{2}\left(\mathbb{R}^{d}\right)$ satisfy

$$
\left(F_{c}, F_{U}, F_{\Sigma}\right) \in \partial F(c, U, \Sigma)
$$

and $E\left[\sup _{t} \mathcal{E}_{t}\left(F_{U}, F_{\Sigma}\right)^{2}\right]<\infty$. Let the process $\pi$ be defined by

$$
\pi=\mathcal{E}\left(F_{U}, F_{\Sigma}\right) F_{c} .
$$

Provided it belongs to $\mathcal{H}$, the process $\pi$ is a supergradient density of $U_{0}$ at $c$. 
Proof. Assuming $c+x \in \mathcal{C}$, we define $\delta=U(c+x)-U(c), \Delta=\Sigma(c+x)-$ $\Sigma(c)$, and $p=F(c, U, \Sigma)+F_{c} x+F_{U} \delta+F_{\Sigma}^{\prime} \Delta-F(c+x, U+\delta, \Sigma+\Delta) \geqslant 0$, where the last inequality follows from the assumed condition (19). The BSDEs for $U(c+h)$ and $U(c)$ imply the linear BSDE

$$
\mathrm{d} \delta=-\left(F_{c} x+F_{U} \delta+F_{\Sigma}^{\prime} \Delta-p\right) \mathrm{d} t+\Delta^{\prime} \mathrm{d} B, \quad \delta_{T}=F_{c}(T) x_{T}-p_{T} .
$$

An exercise, using Lemma 17 and Lemma 5, shows that $\delta_{0} \leqslant(\pi \mid x)$.

\subsection{Optimality under recursive utility}

Proposition 3 verifies the optimality of a feasible consumption plan $c$ based on the existence of a process that is both a utility supergradient density at $c$ and a state price density at $c$. Specializing this argument to recursive utility, in this subsection, we apply Itô's lemma to the supergradient density expression of Proposition 21, and we use the state price dynamics of Proposition 7 to derive sufficient optimality conditions for recursive utility as a FBSDE system.

We fix a reference recursive utility $U: \mathcal{C} \rightarrow I_{U}$ with aggregator $F$, relative to which optimality is defined. By definition, $F(\omega, t, \cdot)$ is concave but not necessarily differentiable. In the following section, we will see that nonsmoothness of $F(\omega, t, c, U, \Sigma)$ in $(U, \Sigma)$ is useful in modeling first-order risk aversion. On the other hand, we will have no use for nonsmoothness of $F$ in the consumption argument, and we therefore assume the existence of the corresponding partial derivative $F_{c}$. In addition, we finesse the issue of a consumption nonnegativity constraint by the usual trick of making marginal utility go to infinity near zero. Finally, we assume that marginal utility converges to zero as consumption goes to infinity. These assumptions and some associated notation are summarized below, and are adopted for the remainder of this chapter's main part.

Regularity assumptions and notation. The partial derivative $F_{c}$ exists everywhere, and the function $F_{c}(\omega, t, \cdot, U, \Sigma)$ is strictly decreasing and maps $(0, \infty)$ onto $(0, \infty)$, for any $(\omega, t, U, \Sigma)$. The function $\mathcal{I}: \Omega \times[0, T] \times(0, \infty) \times I_{U} \times$ $\mathbb{R}^{d} \rightarrow(0, \infty)$ is therefore well-defined implicitly by

$$
F_{c}(\omega, t, \mathcal{I}(\omega, t, \lambda, U, \Sigma), U, \Sigma)=\lambda, \quad \lambda \in(0, \infty) .
$$

The superdifferential of $F$ with respect to $(U, \Sigma)$ is defined by

$$
\begin{aligned}
& \partial_{U, \Sigma} F(\omega, t, c, U, \Sigma) \\
& \quad=\left\{(a, b) \in \mathbb{R} \times \mathbb{R}^{d}:\left(F_{c}(\omega, t, c, U, \Sigma), a, b\right) \in \partial F(\omega, t, c, U, \Sigma)\right\} .
\end{aligned}
$$

We fix a reference strategy $(\rho, \psi)$, generating the wealth process $W$, and financing the consumption plan $c=\rho W$. To formulate sufficient conditions for the optimality of $c$, we define the strictly positive process

$$
\lambda_{t}=F_{c}\left(t, c_{t}, U_{t}, \Sigma_{t}\right) .
$$


The last equation is equivalent to

$$
c_{t}=\mathcal{I}\left(t, \lambda_{t}, U_{t}, \Sigma_{t}\right) \text {. }
$$

By the usual envelope-type argument of microeconomics, if $c$ is optimal then $\lambda_{t}$ represents the shadow price of the time- $t$ wealth constraint. Although we will not need to formalize this interpretation, it will be helpful to keep in mind that $\lambda$ is a shadow-price-of-wealth process. The dynamics of $\lambda$ are denoted

$$
\frac{\mathrm{d} \lambda_{t}}{\lambda_{t}}=\mu_{t}^{\lambda} \mathrm{d} t+\sigma_{t}^{\lambda \prime} \mathrm{d} B_{t} .
$$

We know that (under regularity assumptions) $\pi=\mathcal{E} \lambda$ is a supergradient density at $c$, where $\mathcal{E}=\mathcal{E}\left(F_{U}, F_{\Sigma}\right)$ is computed as in Proposition 21. Integration by parts gives

$$
\frac{\mathrm{d} \pi}{\pi}=\left(F_{U}+\mu^{\lambda}+\sigma^{\lambda \prime} F_{\Sigma}\right) \mathrm{d} t+\left(F_{\Sigma}+\sigma^{\lambda}\right)^{\prime} \mathrm{d} B .
$$

To ensure that $\pi$ is also a state-price density at $c$, we match terms with the dynamics of Proposition 7, resulting in the restrictions:

$$
r=-\left(F_{U}+\mu^{\lambda}+\sigma^{\lambda \prime} F_{\Sigma}\right), \quad \eta=-\left(F_{\Sigma}+\sigma^{\lambda}\right), \quad \mu^{R}=\sigma^{R \prime} \eta .
$$

We round up the optimality conditions by combining the above restrictions with the utility and wealth dynamics, as well as Equations (21) and (22):

Condition 22 (Optimality conditions for recursive utility). The trading strategy $\psi$ and the processes $\left(U, \Sigma, \lambda, \sigma^{\lambda}, W\right) \in \mathcal{U} \times \mathcal{L}_{2}\left(\mathbb{R}^{d}\right) \times \mathcal{L}\left(\mathbb{R}_{++}\right) \times \mathcal{L}_{2}\left(\mathbb{R}^{d}\right) \times$ $\mathcal{L}\left(\mathbb{R}_{++}\right)$solve

$$
\begin{aligned}
& \mathrm{d} U=-F(\mathcal{I}(\lambda, U, \Sigma), U, \Sigma) \mathrm{d} t+\Sigma^{\prime} \mathrm{d} B, \quad U_{T}=F\left(T, W_{T}\right), \\
& \frac{\mathrm{d} \lambda}{\lambda}=-\left(r+F_{U}+\sigma^{\lambda \prime} F_{\Sigma}\right) \mathrm{d} t+\sigma^{\lambda \prime} \mathrm{d} B, \quad \lambda_{T}=F_{c}\left(T, W_{T}\right), \\
& \mathrm{d} W=\left(W\left(r+\psi^{\prime} \mu^{R}\right)-\mathcal{I}(\lambda, U, \Sigma)\right) \mathrm{d} t+W \psi^{\prime} \sigma^{R^{\prime}} \mathrm{d} B, \quad W_{0}=w_{0}, \\
& \mu^{R}+\sigma^{R^{\prime}}\left(F_{\Sigma}+\sigma^{\lambda}\right)=0, \quad\left(F_{U}, F_{\Sigma}\right) \in\left(\partial_{U, \Sigma} F\right)(\mathcal{I}(t, \lambda, U, \Sigma), U, \Sigma) .
\end{aligned}
$$

Proposition 23. Suppose Condition 22 holds, and let $c_{t}=\mathcal{I}\left(t, \lambda_{t}, U_{t}, \Sigma_{t}\right)$ and $\rho_{t}=c_{t} / W_{t}$. If $c \in \mathcal{C}, \pi=\mathcal{E}\left(F_{U}, F_{\Sigma}\right) \lambda \in \mathcal{H}$, and $E\left[\sup _{t} \pi_{t} W_{t}\right]<\infty$, then the strategy $(\rho, \psi)$ is optimal, it generates the wealth process $W$, and it finances the consumption plan $c$, whose utility process is $U$.

Proof. The dynamics of $W$ can be used to verify that $(\rho, \psi)$ finances $c$ with wealth process $W$. By Proposition 21, $\pi$ is a utility supergradient density at $c$. By Proposition 7, $\pi$ is also a state price density at $c$. By Proposition 3, $c$ is optimal. The dynamics of $U$ show that $U=U(c)$. 
Remark 24. In Schroder and Skiadas (2003) the above conditions are extended to include convex trading constraints, and a necessity argument is given for a smooth aggregator under some regularity assumptions. The case of no intermediate or no terminal consumption is essentially the same as above, omitting the appropriate consumption arguments in the formulation.

Condition 22 is a FBSDE system. The wealth dynamics are computed recursively forward in time, starting with $W_{0}=w_{0}$, and the dynamics of $(U, \lambda)$ are computed recursively backward on the information tree, starting with their terminal values. The forward and backward components are coupled. In the following subsection we will introduce scale-invariance as a way of uncoupling this FBSDE system. In a Markovian setting, a PDE version of the FBSDE system can be obtained as in Ma et al. (1994). The construction is outlined in Schroder and Skiadas (2003), as well as later in this chapter for a more special class of homothetic recursive utilities.

\subsection{Homothetic recursive utility}

The utility function $U_{0}: \mathcal{C} \rightarrow \mathbb{R}$ is homothetic (or scale-invariant) if for any $c^{1}, c^{2} \in \mathcal{C}$,

$$
U_{0}\left(c^{1}\right)=U_{0}\left(c^{2}\right) \text { implies } U_{0}\left(k c^{1}\right)=U_{0}\left(k c^{2}\right) \text { for all } k \in(0, \infty) .
$$

If $U_{0}$ is homothetic and cardinal, then ${ }^{12}$ it is homogeneous (of degree one). For recursive utility, with $I_{U}=(0, \infty)$, homogeneity of $U_{0}$ is implied by (and is essentially equivalent to) homogeneity of the aggregator with respect to the utility argument; that is, an aggregator of the form

$$
F(\omega, t, c, U, \Sigma)=U G\left(\omega, t, \frac{c}{U}, \frac{\Sigma}{U}\right), \quad F(\omega, T, c)=c,
$$

for some function $G: \Omega \times[0, T] \times(0, \infty) \times \mathbb{R}^{d} \rightarrow \mathbb{R}$ that we call a proportional aggregator.

Assuming the above aggregator form, suppose that $(\rho, \psi)$ is an optimal strategy with corresponding wealth process $W$ and utility process $U$. Recalling the interpretation of the process $\lambda$ as the sensitivity of the optimal time- $t$ utility value on time- $t$ wealth, the homogeneity of the utility function implies that

$$
U=\lambda W .
$$

The intuition behind this relationship is straightforward. Suppose that at some spot $\omega^{t}$ the investor with unit wealth finds a consumption plan $\bar{c}$ optimal, resulting in the spot- $\omega^{t}$ optimal utility value $\lambda\left[\omega^{t}\right]$. If the same investor's wealth

\footnotetext{
${ }^{12}$ Proof. For any $c \in C$ and $k \in(0, \infty), U_{0}(c)=U_{0}\left(U_{0}(c) \mathbf{1}\right)$ implies $U_{0}(k c)=U_{0}\left(k U_{0}(c) \mathbf{1}\right)=$ $k U_{0}(c)$.
} 
at $\omega^{t}$ were instead $W\left[\omega^{t}\right]$, then, by the homogeneity of the utility function and the budget equation, the investor would find the consumption plan $W\left[\omega^{t}\right] \bar{c}$ optimal at $\omega^{t}$, resulting in the optimal utility value $U\left[\omega^{t}\right]=\lambda\left[\omega^{t}\right] W\left[\omega^{t}\right]$. In other words, the optimization problem at every spot is a scaled version of the unit-wealth version of the problem.

Equation (25) allows us to reduce the optimality conditions to a single BSDE for $\lambda$, whose general form can be found in Schroder and Skiadas (2003). Rather than dealing with the general case here, we will instead consider, in Section 5, optimality under special proportional aggregator functional forms that are motivated by the models of risk aversion of the following section.

We close this section with an example of a proportional aggregator specification, under which the optimal consumption strategy is a given process, for any investment opportunity set.

Example 25 (A robustly optimal consumption strategy). Let the aggregator $F$ be given by Equation (24) for a proportional aggregator of the form

$$
G(t, x, \sigma)=\beta(t) \log (x)+H(t, \sigma), \quad t<T,
$$

where $\beta$ is any strictly positive and (for simplicity) bounded process. While the optimal trading strategy depends on the specification of $H$ and the investment opportunity set, the optimal consumption strategy is independent of both, and is simply equal to $\beta$. Ignoring technical details, we assume the sufficiency and necessity of the optimality conditions and the existence of an optimum. To see the essential part of the argument, suppose $(\rho, \psi)$ is an optimal strategy, with corresponding wealth process $W$, consumption plan $c=\rho W$, utility process $U=\ldots \mathrm{d} t+\Sigma^{\prime} \mathrm{d} B$, and shadow-price-of-wealth process $\lambda$. Using Equation (25), we observe that

$$
\lambda_{t}=F_{c}(t, c, U, \Sigma)=\beta_{t} \frac{U_{t}}{c_{t}}=\beta_{t} \frac{W_{t}}{c_{t}} \frac{U_{t}}{W_{t}}=\frac{\beta_{t}}{\rho_{t}} \lambda_{t} .
$$

Therefore, $\rho=\beta$, independently of the investment opportunity set. [In Schroder and Skiadas (2003) it is shown that this conclusion is valid even under trading constraints.]

\section{Modeling risk aversion}

This section formulates some concrete representations of possibly sourcedependent second- or first-order risk aversion in the context of recursive utility. These representations will be used in the following section to derive optimal trading strategy formulas that help clarify the relationship between risk aversion and optimal portfolio allocations. 


\subsection{Conditional certainty equivalents}

Let us recall the essential intuition of a recursive utility formulation, captured by Lemma 12 . We fix a consumption plan $c$ with cardinal utility process $U=U(c)$. For any time- $t$ spot $\omega^{t}$, the corresponding utility value $U_{t}\left[\omega^{t}\right]$ can be computed as a function of $\omega^{t}$, the immediate consumption $c\left[\omega^{t}\right] \mathrm{d} t$, and the restriction of the random variable $U_{t+\mathrm{d} t}$ to the spot $\omega^{t}$, which we denote $U_{t+\mathrm{d} t}\left[\omega^{t}\right]$.

In this section, we assume that the functional dependence of $U_{t}$ on $U_{t+\mathrm{d} t}$ enters through the conditional certainty equivalent $\nu_{t}\left(U_{t+\mathrm{d} t}\right)$, an $\mathcal{F}_{t}$-measurable random variable such that $\nu_{t}\left(U_{t+\mathrm{d} t}\right)\left[\omega^{t}\right]$ depends on $U_{t+\mathrm{d} t}$ only through its restriction $U_{t+\mathrm{d} t}\left[\omega^{t}\right]$, and is the identity if $U_{t+\mathrm{d} t}\left[\omega^{t}\right]$ is constant. The value of $\nu_{t}\left(U_{t+\mathrm{d} t}\right)$ is a conditional certainty equivalent in the sense that, conditionally on the spot $\omega^{t}$ and immediate consumption $c\left[\omega^{t}\right] \mathrm{d} t$, the investor is indifferent between the continuation of the plan $c$ and a constant consumption rate of $\nu_{t}\left(U_{t+\mathrm{d} t}\right)\left[\omega^{t}\right]$ over the entire remaining period $[t+\mathrm{d} t, T]$. Under this assumption, we can write the heuristic recursion for the utility process $U=U(c)$ as

$$
U_{t}=\phi\left(t, \mathrm{~d} t, c_{t}, \nu_{t}\left(U_{t+\mathrm{d} t}\right)\right),
$$

where $\phi$ can be spot-dependent. The dependence of $\phi$ on the recursion interval $\mathrm{d} t$ is important in the approximation argument that follows. We further assume that $\phi$ has continuous partial derivatives $\phi_{\mathrm{d} t}, \phi_{c}$, and $\phi_{U}$. Since preferences are increasing, $\phi_{c}$ and $\phi_{U}$ are strictly positive.

In the following three subsections we derive the functional form of the aggregator $F$ for various specifications of the certainty equivalent $\nu$. In each case, the conditional certainty equivalent has a local representation in terms of the utility dynamics $\mathrm{d} U_{t}=\mu_{t} \mathrm{~d} t+\Sigma_{t}^{\prime} \mathrm{d} B_{t}$ that takes the form

$$
\nu_{t}\left(U_{t+\mathrm{d} t}\right)=U_{t}+\mu_{t} \mathrm{~d} t-\mathcal{A}\left(t, U_{t}, \Sigma_{t}\right) \mathrm{d} t,
$$

where $\mathcal{A}(t, U, 0)=0$. The function $\mathcal{A}$ represents the risk aversion implicit in $\nu$. Recalling Equations (16) and (27), and using a first-order Taylor expansion of $\phi$, we obtain

$$
\begin{aligned}
U_{t} & =\Phi\left(t, c_{t}, U_{t}+\mu_{t} \mathrm{~d} t, \Sigma_{t}\right) \\
& =\phi\left(t, \mathrm{~d} t, c_{t}, U_{t}+\left(\mu_{t}-\mathcal{A}\left(t, U_{t}, \Sigma_{t}\right)\right) \mathrm{d} t\right) \\
& =U_{t}+\left[\phi_{\mathrm{d} t}\left(t, 0, c_{t}, U_{t}\right)+\phi_{U}\left(t, 0, c_{t}, U_{t}\right)\left(\mu_{t}-\mathcal{A}\left(t, U_{t}, \Sigma_{t}\right)\right)\right] \mathrm{d} t .
\end{aligned}
$$

Using the definition of $F$ in terms of $\Phi$ in (17) and the last equation, we obtain the aggregator functional form

$$
F(\omega, t, c, U, \Sigma)=f(\omega, t, c, U)-\mathcal{A}(\omega, t, U, \Sigma),
$$

where $f(\omega, t, c, U)=\phi_{\mathrm{d} t}(\omega, t, 0, c, U) / \phi_{U}(\omega, t, 0, c, U)$.

Suppose now that $\phi$ and $\mathcal{A}$ are state independent, and therefore $f$ and $F$ are also state independent. If the plan $c$ is deterministic, then $\Sigma=0$ and [since 
$\mathcal{A}(t, U, 0)=0]$ utility can be computed in terms of the aggregator section $F(t, c, U, 0)=f(t, c, U)$. The function $f$ (or $\phi)$ therefore determines the investor's preferences over deterministic choices. By Proposition 20, given $f$, the larger $\mathcal{A}$ is the more risk-averse the investor. This hierarchical separation of preferences toward deterministic choices and risk aversion can also be seen directly in the recursive form (27). If $c$ is deterministic, then so is $U$, and therefore $\nu_{t}\left(U_{t+\mathrm{d} t}\right)=U_{t+\mathrm{d} t}$. This shows that utility over deterministic plans is determined by $\phi$. Given $\phi$, increasing $\mathcal{A}$ decreases the conditional certainty equivalent value and therefore $U_{t}$, resulting in more risk-averse utility.

The key behavioral restriction introduced by the assumption of the recursive form (27) is that, given the agent's preferences over deterministic choices, the agent's risk aversion at a spot $\omega^{t}$, represented by $\mathcal{A}(U, \Sigma)\left[\omega^{t}\right]$, does not depend on the amount $c\left[\omega^{t}\right] \mathrm{d} t$ consumed at time $t$. This separation of current consumption and risk aversion is reflected in the separable representation (29).

Homothetic utility with an aggregator of the form (29) is obtained by further imposing the functional restriction (24). In this case, the proportional aggregator $G$ takes the functional form

$$
G(\omega, t, x, \sigma)=g(\omega, t, x)-\mathcal{R}(\omega, t, \sigma),
$$

where $g(\omega, t, x)=f(\omega, t, x, 1)$ and $\mathcal{R}(\omega, t, \sigma)=\mathcal{A}(\omega, t, 1, \sigma)$.

\subsection{The Duffie-Epstein limit of Kreps-Porteus utility}

The first specialization of the aggregator form (29) we consider results from the continuous-time formulation of Kreps and Porteus (1978) utility due to ${ }^{13}$ Duffie and Epstein (1992). In this formulation, the conditional certainty equivalent $\nu_{t}$ is defined by

$$
u\left(\nu_{t}\left(U_{t+\mathrm{d} t}\right)\right)=E_{t}\left[u\left(U_{t+\mathrm{d} t}\right)\right],
$$

for some strictly increasing, concave, twice continuously differentiable function $u: I_{U} \rightarrow \mathbb{R}$. We denote the corresponding coefficient of absolute risk aversion by

$$
a(U)=-\frac{u^{\prime \prime}(U)}{u^{\prime}(U)} .
$$

In the current context, the classic Arrow $(1965,1970)$ and Pratt (1964) approximation of expected utility for small risks can be expressed through Itô's

\footnotetext{
${ }^{13}$ In fact, Duffie-Epstein utilities are obtained as the continuous-time limit of a broader class of discrete-time utilities than the Kreps-Porteus class, since the investor's certainty equivalent over continuation utility need only be von Neumann-Morgenstern in an approximate local sense. It is sufficient for our purposes, however, to think of Duffie-Epstein utility as (sufficiently smooth) continuous-time Kreps-Porteus utility.
} 
lemma as

$$
u\left(U_{t+\mathrm{d} t}\right)=u\left(U_{t}\right)+u^{\prime}\left(U_{t}\right) \mathrm{d} U_{t}+\frac{1}{2} u^{\prime \prime}\left(U_{t}\right)\left(\mathrm{d} U_{t}\right)^{2} .
$$

Given the utility process Itô decomposition $\mathrm{d} U_{t}=\mu_{t} \mathrm{~d} t+\Sigma_{t}^{\prime} \mathrm{d} B_{t}$, the above results in

$$
E_{t}\left[u\left(U_{t+\mathrm{d} t}\right)\right]=u\left(U_{t}\right)+u^{\prime}\left(U_{t}\right) \mu_{t} \mathrm{~d} t+\frac{1}{2} u^{\prime \prime}\left(U_{t}\right) \Sigma_{t}^{\prime} \Sigma_{t} \mathrm{~d} t .
$$

On the other hand, a first-order Taylor expansion gives

$$
u\left(\nu_{t}\left(U_{t+\mathrm{d} t}\right)\right)=u\left(U_{t}\right)+u^{\prime}\left(U_{t}\right)\left(\nu_{t}\left(U_{t+\mathrm{d} t}\right)-U_{t}\right) .
$$

Combining the last two equations with the certainty equivalent definition (31) results in the certainty-equivalent expression (28) with the quadratic riskaversion component

$$
\mathcal{A}(\omega, t, U, \Sigma)=\frac{1}{2} a(U) \Sigma^{\prime} \Sigma .
$$

The corresponding aggregator (29) takes Duffie-Epstein form:

$$
F(\omega, t, c, U, \Sigma)=f(\omega, t, c, U)-\frac{1}{2} a(U) \Sigma^{\prime} \Sigma .
$$

We refer to Duffie and Epstein (1992) for further analysis of this utility form. For example, they show that there is always an ordinally equivalent utility version with the same recursive representation but $a=0$. The latter restriction can be analytically helpful, but minimizes the usefulness of the hierarchical separation of choice over deterministic plans and risk aversion of Proposition 20. If $a=0$ and $F=f$ is linear in $U$, as in Example 13, then one obtains timeadditive expected discounted utility.

Homothetic Duffie-Epstein utility is obtained if the aggregator takes the homogeneous form (24), for a proportional aggregator of the form (30) with $\mathcal{R}(\omega, t, \sigma)=(\gamma / 2) \sigma^{\prime} \sigma$ for some $\gamma \in \mathbb{R}_{+}$. In this case, the BSDE specifying the utility process $U=U(c)$ is

$$
\frac{\mathrm{d} U_{t}}{U_{t}}=-\left(g\left(t, \frac{c_{t}}{U_{t}}\right)-\frac{\gamma}{2} \sigma_{t}^{\prime} \sigma_{t}\right) \mathrm{d} t+\sigma_{t}^{\prime} \mathrm{d} B_{t}, \quad U_{T}=c_{T} .
$$

The coefficient $\gamma$ can be obtained from the certainty equivalent (31) with

$$
u(U)=\frac{U^{1-\gamma}-1}{1-\gamma}
$$

in which case $\gamma=a(U) U$ is the coefficient of relative risk aversion of the von Neumann-Morgenstern utility $u$. Here and below, we interpret the function (34) with $\gamma=1$ by taking the limit as $\gamma \rightarrow 1$, resulting in $u(U)=$ $\log U$. Assuming it is state-independent, the function $g$ entirely determines the agent's preferences over deterministic consumption plans. Given $g$, increasing the value of $\gamma$ makes the agent more risk averse. 
Example 26. The continuous-time version of Epstein-Zin utility is contained in the specification

$$
\begin{aligned}
& g(\omega, t, x)=\alpha+\beta \frac{x^{1-\delta}-1}{1-\delta}, \\
& \quad \text { where } \alpha \in \mathbb{R}, \beta \in(0, \infty), \delta \in[0, \infty) .
\end{aligned}
$$

Within this class, the utility is additive if and only if $\gamma=\delta$, a condition that ties the relative risk-aversion coefficient $\gamma$ to a parameter that is determined entirely by the agent's preferences over deterministic plans. Assuming $\gamma=\delta$, let $b=\beta-(1-\gamma) \alpha$, and consider the ordinally equivalent utility process

$$
V_{t}(c)=\frac{1}{\beta} \frac{U_{t}(c)^{1-\gamma}-1}{1-\gamma}-\frac{\alpha}{\beta} \int_{t}^{T} e^{-b(s-t)} \mathrm{d} s .
$$

An exercise using Itô's lemma shows that ${ }^{14}$

$$
V_{t}(c)=E_{t}\left[\int_{t}^{T} e^{-b(s-t)} \frac{c_{s}^{1-\gamma}-1}{1-\gamma} \mathrm{d} s+\frac{1}{\beta} e^{-b(T-t)} \frac{c_{T}^{1-\gamma}-1}{1-\gamma}\right] .
$$

\subsection{Source-dependent risk aversion}

As noted in the Introduction, it is of interest to consider risk aversion that can depend on the source of risk, for example, as an expression of aversion to ambiguity associated with a given source of risk. With a version of Proposition 20, Lazrak and Quenez (2003) made the important observation that the functional dependence of a general aggregator $F(t, c, U, \Sigma)$ on $\Sigma$ allows the modeling of risk-aversion that varies with the direction of risk. Since $\Sigma$ represents loadings to instantaneous linear factors, such directional risk aversion can be interpreted as source-dependent risk aversion. In this section and the following one, we motivate some special functional aggregator forms representing source-dependent risk aversion that were introduced in Schroder and Skiadas (2003) (for the homothetic case).

We begin with a simple extension of Duffie-Epstein utility that allows for source-dependent risk aversion, where each Brownian motion is viewed as a separate source of risk. In the Duffie-Epstein formulation, the certainty equivalent (31) is applied to the continuation utility, $U_{t+\mathrm{d} t}=U_{t}+\mu_{t} \mathrm{~d} t+\Sigma_{t}^{\prime} \mathrm{d} B_{t}$, which aggregates all sources of risk. Here we assume that the investor perceives and worries about the individual risk terms $\Sigma_{t}^{1} \mathrm{~d} B_{t}^{1}, \ldots, \Sigma_{t}^{d} \mathrm{~d} B_{t}^{d}$ separately,

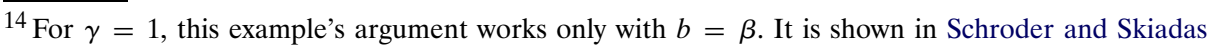
(2005b), however, that any $V$ of the form (36) is ordinally equivalent to a homothetic Duffie-Epstein utility (33), with $g$ specified as in (35) with $\alpha=0$ and $\beta$ a deterministic function of time (even if $\gamma=1$ and $b \neq \beta)$.
} 
since they represent exposure to different sources of risk. We model this by postulating a twice continuously differentiable concave function $u: \mathbb{R}^{1+d} \rightarrow \mathbb{R}$ such that the time- $t$ conditional certainty equivalent in the recursive specification (27) is defined by

$$
u\left(\nu_{t}\left(U_{t+\mathrm{d} t}\right), 0, \ldots, 0\right)=E_{t}\left[u\left(U_{t}+\mu_{t} \mathrm{~d} t, \Sigma^{1} \mathrm{~d} B^{1}, \ldots, \Sigma^{d} \mathrm{~d} B^{d}\right)\right] .
$$

The first- and second-order partial derivatives of $u\left(x_{0}, x_{1}, \ldots, x_{d}\right)$ with respect to $x_{i}$ are denoted $u_{i}$ and $u_{i i}$, respectively. We assume that $u$ is strictly increasing in its first argument. The absolute risk aversion coefficient with respect to the $i$ th risk source is defined by

$$
a^{i}(U)=-\frac{u_{i i}(U, 0, \ldots, 0)}{u_{0}(U, 0, \ldots, 0)} .
$$

We also define the diagonal matrix $A(U)=\operatorname{diag}\left[a^{1}(U), \ldots, a^{d}(U)\right]$. Applying Itô's lemma and taking conditional expectations results in

$$
\begin{aligned}
E_{t} & {\left[u\left(U_{t}+\mu_{t} \mathrm{~d} t, \Sigma^{1} \mathrm{~d} B^{1}, \ldots, \Sigma^{d} \mathrm{~d} B^{d}\right)\right] } \\
& =u\left(U_{t}, 0, \ldots, 0\right)+u_{0}\left(U_{t}, 0, \ldots, 0\right)\left(\mu_{t}-\frac{1}{2} \Sigma_{t}^{\prime} A\left(U_{t}\right) \Sigma_{t}\right) \mathrm{d} t .
\end{aligned}
$$

Similarly, we have the first-order Taylor expansion

$$
\begin{aligned}
u\left(\nu_{t}\left(U_{t+\mathrm{d} t}\right), 0, \ldots, 0\right)= & u\left(U_{t}, 0, \ldots, 0\right)+u_{0}\left(U_{t}, 0, \ldots, 0\right) \\
& \times\left(\nu_{t}\left(U_{t+\mathrm{d} t}\right)-U_{t}\right) .
\end{aligned}
$$

Matching the lasts two expressions and simplifying results in the certaintyequivalent expression (28), and corresponding aggregator (29), with the quadratic risk-aversion component

$$
\mathcal{A}(\omega, t, U, \Sigma)=\frac{1}{2} \Sigma^{\prime} A(U) \Sigma .
$$

The Duffie-Epstein case is obtained if $a^{i}=a$ for all $i$. Combining the above representation with the homothetic specification (24) results in a proportional aggregator of the form (30), where $\mathcal{R}$ is a quadratic form.

Remark 27. A simple extension is obtained if the Brownian motion in the above argument is replaced by a new Brownian motion $\bar{B}$, where $\mathrm{d} \bar{B}$ is a possibly spot-dependent rotation of $\mathrm{d} B$. More formally, we assume $\mathrm{d} \bar{B}_{t}=\Phi_{t} \mathrm{~d} B_{t}$ for some $\Phi \in \mathcal{L}_{2}\left(\mathbb{R}^{d \times d}\right)$ such that $\Phi_{t}^{\prime} \Phi_{t}=I$. In this case, $U_{t+\mathrm{d} t}=U_{t}+\mu_{t} \mathrm{~d} t+$ $\bar{\Sigma}_{t}^{\prime} \mathrm{d} \bar{B}_{t}$, where $\bar{\Sigma}_{t}=\Phi_{t} \Sigma_{t}$, and

$$
\mathcal{A}\left(t, U_{t}, \Sigma_{t}\right)=\frac{1}{2} \bar{\Sigma}_{t}^{\prime} A\left(U_{t}\right) \bar{\Sigma}_{t}=\frac{1}{2} \Sigma_{t}^{\prime} \Phi_{t}^{\prime} A\left(U_{t}\right) \Phi_{t} \Sigma_{t} .
$$

In the Duffie-Epstein case, $\Phi^{\prime} A \Phi=A$. With source-dependent risk aversion, however, the aggregator form changes with the Brownian motion rotation. 


\subsection{First-order risk aversion}

Consider an investor who maximizes expected von Neumann-Morgenstern (vNM) utility in a single-period setting. If one were to zoom in a very small area of the graph of the vNM utility, one would see a straight line. This means that an investor is essentially risk-neutral toward the addition of sufficiently small risks to a given wealth level. As an implication, such an investor would seek some exposure to all investment opportunities of positive expected excess return, and would not completely insure a source of risk in actuarially unfavorable terms. These conclusions extend to the recursive utility formulations of the last two sections, as will become clear in the following section. In reality, we observe that investors do not necessarily participate in investment opportunities with positive Sharpe ratios, and they often pay actuarially unfavorable premia to completely insure some sources of risk (for example, against loss of individual items of negligible value relative to total wealth). While such behavior can relate to a number of issues, it is consistent with a certainty-equivalent specification exhibiting first-order risk aversion in the sense of Segal and Spivak (1990). In this subsection, we formulate recursive utility with a conditional certainty equivalent exhibiting first-order risk aversion, whose implication for portfolio choice is discussed in the following section.

In a static expected-utility setting, first-order risk aversion amounts to introducing a kink of the vNM utility around the given wealth level, hence removing local risk neutrality. Since a risk-averse vNM utility can have at most countably many kinks, the approach seems problematic. If one keeps track of different sources of risk, however, as in the source-dependent certainty equivalent introduced above, this problem does not arise. As in the last subsection, we assume the recursive utility specification (27) with the source-dependent certainty equivalent specification in (37), except that the function $u$ in (37) is now replaced with the function

$$
\hat{u}\left(x_{0}, x_{1}, \ldots, x_{d}\right)=u\left(x_{0}, x_{1}, \ldots, x_{d}\right)-\sum_{i=1}^{d} \delta^{i}\left(x_{0}\right)\left|x_{i}\right| \sqrt{\mathrm{d} t} .
$$

The $\sqrt{\mathrm{d} t}$ scaling factor is necessary for a meaningful trade-off between the conditional mean of $\mathrm{d} U_{t}$, which is order $\mathrm{d} t$, and the conditional absolute variation of $\Sigma^{i} \mathrm{~d} B^{i}$, which is order $\sqrt{\mathrm{d} t}$. We assume that each $\delta^{i}$ is differentiable and nonnegative valued, and that $u$ is exactly as in the last subsection. Since $\hat{u}(U, 0, \ldots, 0)=u(U, 0, \ldots 0)$, the conditional certainty equivalent $\nu_{t}$ is specified by

$$
\begin{aligned}
u\left(\nu_{t}\left(U_{t+\mathrm{d} t}\right), 0, \ldots, 0\right)= & E_{t}\left[u\left(U_{t}+\mu_{t} \mathrm{~d} t, \Sigma^{1} \mathrm{~d} B^{1}, \ldots, \Sigma^{d} \mathrm{~d} B^{d}\right)\right] \\
& -\sum_{i=1}^{d} E_{t}\left[\delta^{i}\left(U_{t}+\mu_{t} \mathrm{~d} t\right)\left|\Sigma_{t}^{i} \mathrm{~d} B_{t}^{i}\right| \sqrt{\mathrm{d} t}\right] .
\end{aligned}
$$


The left-hand side and the first term of the right-hand side in the above equation are computed exactly as in the last subsection. To compute the last term, we first note that $E_{t}\left|\mathrm{~d} B_{t}^{i}\right|=\sqrt{2 \mathrm{~d} t / \pi}$ (since $\mathrm{d} B_{t}^{i}$ is normally distributed with zero mean and variance $\mathrm{d} t)$. Using the first-order Taylor expansion $\delta^{i}\left(U_{t}+\right.$ $\left.\mu_{t} \mathrm{~d} t\right)=\delta^{i}\left(U_{t}\right)+\delta^{i \prime}\left(U_{t}\right) \mu_{t} \mathrm{~d} t$ and the usual Itô calculus, we find

$$
E_{t}\left[\delta^{i}\left(U_{t}+\mu_{t} \mathrm{~d} t\right)\left|\Sigma_{t}^{i} \mathrm{~d} B_{t}^{i}\right| \sqrt{\mathrm{d} t}\right]=u_{0}(U, 0, \ldots, 0) \kappa^{i}\left(U_{t}\right)\left|\Sigma_{t}^{i}\right| \mathrm{d} t,
$$

where $\kappa^{i}(U)=\sqrt{2 / \pi} \delta^{i}(U) / u_{0}(U, 0, \ldots, 0)$. Substituting these calculations in the above equation specifying $\nu_{t}$ results in the conditional certainty equivalent expression (28), and corresponding aggregator (29), with

$$
\begin{aligned}
\mathcal{A}(\omega, t, U, \Sigma) & =\kappa(U)^{\prime}|\Sigma|+\frac{1}{2} \Sigma^{\prime} A(U) \Sigma, \\
\text { where } \kappa(U) & =\left(\kappa^{1}(U), \ldots, \kappa^{d}(U)\right)^{\prime} \text { and }|\Sigma|=\left(\left|\Sigma^{1}\right|, \ldots,\left|\Sigma^{d}\right|\right)^{\prime} .
\end{aligned}
$$

For the homothetic specification (24), the proportional aggregator takes the form (30), where $\mathcal{R}(\omega, t, \sigma)=\kappa(1)^{\prime}|\Sigma|+(1 / 2) \Sigma^{\prime} A(1) \Sigma$. We revisit the homothetic case in the following section, where the effect of first-order risk aversion on portfolio choice is discussed.

A dual formulation of this subsection's utility corresponds to the " $\kappa$ ignorance" multiple-prior formulation of Chen and Epstein (2002). Further discussion of recursive utility duality can be found in El Karoui et al. (2001).

\section{Scale-invariant solutions}

In this section we study optimal strategies under the homothetic case of last section's utilities, thus taking advantage of the simplifications of scale invariance introduced in Section 3.4, as well as specific risk-aversion parameterizations.

The following condition is assumed to hold throughout the section.

Condition 28. Utility processes are valued in $I_{U}=(0, \infty)$, and are defined in terms of the functions ${ }^{15} g:[0, T] \times(0, \infty) \rightarrow \mathbb{R}$ and $\mathcal{R}: \Omega \times[0, T] \times \mathbb{R}^{d} \rightarrow \mathbb{R}$, where $\mathcal{R}(\omega, t, 0)=0$. For any $c \in \mathcal{C}$, the utility process $U=U(c)$ solves, uniquely in $\mathcal{U}$, the BSDE

$$
\frac{\mathrm{d} U_{t}}{U_{t}}=-\left(g\left(t, \frac{c_{t}}{U_{t}}\right)-\mathcal{R}\left(t, \sigma_{t}\right)\right) \mathrm{d} t+\sigma_{t}^{\prime} \mathrm{d} B_{t}, \quad U_{T}=c_{T} .
$$

For every time $t, g(t, \cdot)$ is differentiable and strictly concave, with derivative $g_{x}(t, \cdot)$ that maps $(0, \infty)$ onto $(0, \infty)$. Finally, $g$ is sufficiently regular so

\footnotetext{
${ }^{15}$ The function $g$ is assumed state-independent for economy of exposition. The optimality conditions, however, remain valid for a state-dependent $g$.
} 
that, for any deterministic $c \in \mathcal{C}$, the ordinary differential equation $\mathrm{d} U / U=$ $-g(t, c / U) \mathrm{d} t, U_{T}=1$, has a unique deterministic solution $U$ in $\mathcal{U}$.

The function $g$ determines choice over deterministic plans. Given $g$, increasing $\mathcal{R}$ increases the investor's risk aversion, without changing the investor's preferences over deterministic plans. The restriction on $g_{x}$ reflects our earlier assumption on $F_{c}$, and guarantees the strict positivity of an optimal consumption plan.

To state the simplified optimality conditions under the above specification, we introduce some notation. The functions $\mathcal{I}^{g}, g^{*}:[0, T] \times(0, \infty) \rightarrow(0, \infty)$ are defined by

$$
\begin{aligned}
& g_{x}\left(t, \mathcal{I}^{g}(t, \lambda)\right)=\lambda \text { and } \\
& g^{*}(t, \lambda)=\max _{x \in \mathbb{R}_{++}}(g(t, x)-\lambda x)=g\left(t, \mathcal{I}^{g}(t, \lambda)\right)-\mathcal{I}^{g}(t, \lambda) \lambda .
\end{aligned}
$$

For any strictly positive Itô process $y$ (such as $\lambda, U$, or $W$ ), the notation $\sigma^{y}$ is defined by the Itô decomposition

$$
\frac{\mathrm{d} y}{y}=\ldots \mathrm{d} t+\sigma^{y^{\prime}} \mathrm{d} B .
$$

As discussed in Section 3.4, since utility is homogeneous of degree-one, at the optimum, the utility process $U$, the wealth process $W$, and the shadowprice-of-wealth process $\lambda$ are related by $U=\lambda W$. The central part of the optimality conditions to follow will be a BSDE solved by $\left(\lambda, \sigma^{\lambda}\right)$. The form of this BSDE is specified by the utility parameters $(g, \mathcal{R})$, and the investment opportunity set parameters $\left(r, \mu^{R}, \sigma^{R}\right)$. The optimal strategy $(\rho, \psi)$ is computed in terms of $\left(\lambda, \sigma^{\lambda}\right)$ by simple formulas. For the optimal consumption strategy, we note that

$$
\rho=\frac{c}{W}=\frac{U}{W} \frac{c}{U}=\lambda x, \quad \text { where } x=\frac{c}{U} .
$$

Since $\lambda=F_{c}(t, c, U, \Sigma)=g_{x}(t, x)$, it follows that

$$
\rho_{t}=\lambda_{t} \mathcal{I}^{g}\left(t, \lambda_{t}\right) .
$$

The optimal consumption strategy is therefore determined entirely by $\lambda$ and $g$. On the other hand, for last section's risk-aversion models, we will see that the optimal trading strategy $\psi$ is determined entirely by $\sigma^{\lambda}$, the risk-aversion function $\mathcal{R}$, and the excess-return parameters $\left(\mu^{R}, \sigma^{R}\right)$.

\subsection{Smooth quasi-quadratic proportional aggregator}

The first specification we consider includes the homothetic version of last section's models of, possibly source-dependent, risk aversion with a smooth aggregator. The case of first-order risk aversion will be treated at the end of this section. Up to that point, we assume: 
Condition 29 (Smooth quasi-quadratic proportional aggregator). Condition 28 holds with

$$
\mathcal{R}(\omega, t, \sigma)=\frac{1}{2} \sigma^{\prime} Q(\omega, t) \sigma,
$$

for some bounded $Q: \Omega \times[0, T] \rightarrow \mathbb{R}^{d \times d}$, where $Q(\omega, t)$ is symmetric positive definite for all $(\omega, t)$.

In terms of the risk-aversion function $A(U)$ of Section 4.3, $Q=A(1)$, and therefore $Q$ can be thought of as a relative risk-aversion process. In the DuffieEpstein case, $Q=\gamma I$, where $\gamma$ is a coefficient of relative risk aversion, common to all sources of risk. If $Q$ is diagonal, then its $i$ th diagonal element corresponds to relative risk aversion toward risk generated by the $i$ th Brownian motion. Remark 27 leads us to consider nondiagonal positive definite specifications of $Q$. In last section's parametric formulations of risk aversion, the conditional certainty equivalent was defined in a spot-independent way in terms of the function $u$, resulting in $Q$ being constant. The same analysis goes through, however, for a function $u$, and associated conditional certainty equivalent, that is spot-dependent, implying a stochastic risk-aversion process $Q$.

For every $(\omega, t) \in \Omega \times[0, T]$, the quadratic function $\mathcal{Q}(\omega, t, \cdot): \mathbb{R}^{d} \rightarrow \mathbb{R}$ is defined by

$$
\begin{aligned}
\mathcal{Q}(t, z)= & z^{\prime} Q_{t} z-\left(\mu_{t}^{R}-\sigma_{t}^{R^{\prime}}\left(Q_{t}-I\right) z\right)^{\prime}\left(\sigma_{t}^{R^{\prime}} Q_{t} \sigma_{t}^{R}\right)^{-1} \\
& \times\left(\mu_{t}^{R}-\sigma_{t}^{R^{\prime}}\left(Q_{t}-I\right) z\right), \quad z \in \mathbb{R}^{d} .
\end{aligned}
$$

Under Condition 29, the solution of the optimality conditions (stated in Condition 22) reduces to the following procedure:

1. Compute $\left(\lambda, \sigma^{\lambda}\right)$ by solving the BSDE:

$$
\frac{\mathrm{d} \lambda_{t}}{\lambda_{t}}=-\left(r_{t}+g^{*}(t, \lambda)-\frac{1}{2} \mathcal{Q}\left(t, \sigma^{\lambda}\right)\right) \mathrm{d} t+\sigma_{t}^{\lambda \prime} \mathrm{d} B_{t}, \quad \lambda_{T}=1 .
$$

2. Given the solution $\left(\lambda, \sigma^{\lambda}\right)$, the optimal consumption strategy $\rho$ is given by Equation (40), and the optimal trading strategy is

$$
\psi_{t}=\left(\sigma_{t}^{R \prime} Q_{t} \sigma_{t}^{R}\right)^{-1}\left(\mu_{t}^{R}-\sigma_{t}^{R^{\prime}}\left(Q_{t}-I\right) \sigma_{t}^{\lambda}\right) .
$$

3. The wealth process $W$ generated by the strategy $(\rho, \psi)$ is computed from the budget equation (4). The optimal consumption plan financed by $(\rho, \psi)$ is $c=\rho W$, and the utility process of $c$ is $U=\lambda W$.

The proof of this claim (given in Schroder and Skiadas, 2003) is a matter of direct calculation using the specific aggregator form, and the key homogeneity condition $U=\lambda W$. The optimal trading strategy expression follows from Equations (23), which imply that $\mu^{R}+\sigma^{R \prime}\left(F_{\Sigma}+\sigma^{\lambda}\right)=0$. In this equation we substitute $F_{\Sigma}=-Q \sigma^{U}$ (from the definition of $F$ ), $\sigma^{U}=\sigma^{\lambda}+\sigma^{W}$ 
(from $U=\lambda W$ ), and $\sigma^{W}=\sigma^{R} \psi$ (from the budget equation). Solving for $\psi$ gives (43).

The optimal trading strategy of Equation (43) can deviate from an instantaneously mean-variance efficient solution for two possible reasons. One is source dependence of risk aversion, reflected in $Q$, and the other is the term involving $\sigma^{\lambda}$ which arises from a stochastic investment opportunity set or stochastic risk aversion. Two special cases in which instantaneous mean-variance efficiency is recovered are given in the following examples. For expositional simplicity, we informally identify optimality with the above optimality conditions (ignoring the regularity assumptions required for actual equivalence).

Example 30 (Deterministic investment opportunity set and risk aversion). Suppose that the investment opportunity set parameters $\left(r, \mu^{R}, \sigma^{R}\right)$, and the risk-aversion process $Q$ are all deterministic. Then the solution simplifies significantly by setting $\sigma^{\lambda}=0$. That is, $\lambda$ is a deterministic process solving the ODE

$$
\frac{\mathrm{d} \lambda_{t}}{\lambda_{t}}=-\left(r_{t}+g^{*}(t, \lambda)+\frac{1}{2} \mu_{t}^{R \prime}\left(\sigma_{t}^{R \prime} Q_{t} \sigma_{t}^{R}\right)^{-1} \mu_{t}^{R}\right) \mathrm{d} t, \quad \lambda_{T}=1 .
$$

Since $\lambda$ is deterministic and $g$ is assumed state-independent, the optimal consumption strategy $\rho=\lambda \mathcal{I}^{g}(\lambda)$ is also deterministic. The optimal trading strategy is $\psi=\left(\sigma^{R \prime} Q \sigma^{R}\right)^{-1} \mu^{R}$.

Suppose further that risk aversion is source-independent, and therefore $Q=\gamma I$ for some deterministic process $\gamma$. Then the optimal trading strategy $\psi=\gamma^{-1}\left(\sigma^{R \prime} \sigma^{R}\right)^{-1} \mu^{R}$ is instantaneously mean-variance efficient. Since $\psi$ does not depend on $g$, it is the same as for the choice of $g$ given in Example 26 with $\gamma=\delta$. In other words, the optimal trading strategy is the same as for time-additive power expected utility (considered by Merton, 1971). On the other hand, $\lambda$ and the optimal consumption strategy depend on the specification of $g$. It is also worth noting that in the current special context the investment opportunity set enters the dynamics of $\lambda$ only through the maximum squared conditional Sharpe ratio of Equation (3).

Example 31 (Robustly mean-variance efficient optimal trading strategies). Even under a stochastic investment opportunity set, the instantaneously meanvariance efficient strategy $\psi=\left(\sigma^{R \prime} \sigma^{R}\right)^{-1} \mu^{R}$ is optimal if $Q=I$ (the identity matrix). Moreover, for $Q=I$, the investment opportunity set enters the BSDE for $\lambda$ only through $\lambda$ and the maximum squared instantaneous Sharpe ratio of Equation (3). Combining time additivity with the assumption $Q=I$ implies that $g$ is logarithmic (Example 26 with $\gamma=\delta=1$ ), and therefore the optimal consumption strategy equals the utility discount rate as in Example 25. Without time-additivity, $g$ is unrestricted. A discrete-time example of this type was first given by Giovannini and Weil (1989). The construction is further extended in Example 33 below. 
As noted earlier, in a Markovian setting, a BSDE is characterized (under some regularity) by a corresponding PDE. The argument is outlined below for the BSDE (42) satisfied by $\lambda$.

Example 32 (Markovian solutions). Given is some underlying $n$-dimensional Markov process $Z$, uniquely solving the SDE

$$
\mathrm{d} Z=a(t, Z) \mathrm{d} t+b(t, Z)^{\prime} \mathrm{d} B, \quad Z_{0}=z_{0},
$$

for some $z_{0} \in \mathbb{R}^{n}$ and functions $a:[0, T] \times \mathbb{R}^{n} \rightarrow \mathbb{R}^{n}$ and $b:[0, T] \times \mathbb{R}^{n} \rightarrow$ $\mathbb{R}^{d \times n}$. With some convenient abuse of notation, we assume that

$$
r_{t}=r\left(t, Z_{t}\right) \quad \text { and } \quad \eta_{t}=\eta\left(t, Z_{t}\right),
$$

for some functions $r:[0, T] \times \mathbb{R}^{n} \rightarrow \mathbb{R}$ and $\eta:[0, T] \times \mathbb{R}^{n} \rightarrow \mathbb{R}^{d}$. We conjecture that $\lambda$ can be written as a function of time and the Markov state that is smooth enough to apply Itô's lemma. With the usual abuse of notation, we write $\lambda(\omega, t)=\lambda(t, Z(\omega, t))$. Omitting the argument $\left(t, Z_{t}\right)$, and with subscripts of $\lambda$ denoting partial derivatives, Itô's lemma implies:

$$
\mathrm{d} \lambda=\left(\lambda_{t}+\lambda_{z}^{\prime} a+\frac{1}{2} \operatorname{tr}\left[b \lambda_{z z} b^{\prime}\right]\right) \mathrm{d} t+\left(b \lambda_{z}\right)^{\prime} \mathrm{d} B .
$$

Comparing the above dynamics to BSDE (42) suggests that $\sigma^{\lambda}=b \lambda_{z} / \lambda$ and $\lambda$ solves the PDE

$$
\begin{gathered}
r+g^{*}(\lambda)-\frac{1}{2} \mathcal{Q}\left(b \frac{\lambda_{z}}{\lambda}\right)+\frac{\lambda_{t}}{\lambda}+a^{\prime} \frac{\lambda_{z}}{\lambda}+\frac{1}{2} \operatorname{tr}\left[b \frac{\lambda_{z z}}{\lambda} b^{\prime}\right]=0, \\
\lambda(T, \cdot)=1,
\end{gathered}
$$

where $r$ and $\mathcal{Q}$ are viewed as functions of the underlying Markov state, in the same notational pattern used earlier for $\lambda$. Reversing the above steps, one can construct a solution to BSDE (42) from a solution to the above PDE.

\subsection{Relating complete and incomplete market solutions}

Continuing with the assumption of a smooth quasiquadratic proportional aggregator (Condition 29), we consider some connections between complete and incomplete market solutions. (The type of market incompleteness discussed here leaves out the case of undiversifiable income risk. A tractable class of problems dealing with the latter is outlined in the final section.)

We recall that $m$ is the number of traded risky assets. Given a matrix $A$ of dimension $n \times k$, where $n \geqslant m$, we use the block matrix notation:

$$
A=\left[\begin{array}{c}
A_{M} \\
A_{N}
\end{array}\right], \quad A_{M} \in \mathbb{R}^{m \times k}, A_{N} \in \mathbb{R}^{(n-m) \times k} .
$$

In particular, $R=R_{M}, \mu^{R}=\mu_{M}^{R}$, and $\sigma^{R \prime}=\left[\sigma_{M}^{R \prime}, \sigma_{N}^{R \prime}\right]$. 
While the solution summarized by BSDE (42) is valid for incomplete markets, the role of nonmarketed uncertainty becomes clearer after passing to a new Brownian motion that generates the same filtration as $B$, and separates marketed and nonmarketed uncertainty. Informally, at each spot, the linear span of $\mathrm{d} R_{M}-\mu_{M}^{R} \mathrm{~d} t$ can be obtained as the linear span of the first $m$ elements of a rotated version of $\mathrm{d} B$. This transformation (stated formally in Schroder and Skiadas, 2003) corresponds to the type of spot-by-spot Brownian motion rotation of Remark 27, which preserves the quasiquadratic proportional aggregator structure. We therefore lose no generality in assuming that

$$
\mathrm{d} R_{M}=\mu_{M}^{R} \mathrm{~d} t+\sigma_{M}^{R \prime} \mathrm{d} B_{M} \quad \text { and } \quad \sigma_{N}^{R}=0 .
$$

For the remainder of this section, we assume the normalized return structure (44), and we think of $M$ and $N$ as sets of indices corresponding to marketed and nonmarketed uncertainty, respectively. The processes $r, \mu_{M}^{R}$, and $\sigma_{M}^{R}$ need not, however, be adapted to the filtration generated by $B_{M}$.

A market-price-of-risk process in this context takes the form

$$
\eta=\left[\begin{array}{l}
\eta_{M} \\
\eta_{N}
\end{array}\right], \quad \text { where } \eta_{M}=\left(\sigma_{M}^{R \prime}\right)^{-1} \mu_{M}^{R} .
$$

The process $\eta_{M}$ represents the price of marketed risk, while the unrestricted process $\eta_{N}$ represents the price of nonmarketed risk. The latter parameterizes the set of every state price density $\pi$ consistent with the given market:

$$
\begin{gathered}
\pi=\pi^{M} \xi^{\eta_{N}}, \quad \text { where } \frac{\mathrm{d} \pi^{M}}{\pi^{M}}=-r \mathrm{~d} t-\eta_{M}^{\prime} \mathrm{d} B_{M} \\
\text { and } \frac{\mathrm{d} \xi^{\eta_{N}}}{\xi^{\eta_{N}}}=-\eta_{N}^{\prime} \mathrm{d} B_{N} .
\end{gathered}
$$

If $\pi$ is a state price density that is also a utility supergradient density at an optimum, then the corresponding $\eta_{N}$ reflects the shadow price of nonmarketed risk, in the following sense. Consider a hypothetical market completion in which risk generated by $\mathrm{d} B_{N}$ is priced by $\eta_{N}$. In such a market, the investor would find it optimal to not trade risk generated by $\mathrm{d} B_{N}$, since the original incomplete-markets strategy would still be optimal. Since the original strategy need not be optimal under any other choice of $\eta_{N}$, the incomplete-markets optimal utility is the minimum of optimal utilities over all market completions (parameterized by $\eta_{N}$ ). This connection between complete and incomplete market solutions is illustrated more concretely in Example 34 below, and extends to more general convex constraints (see Cvitanić and Karatzas, 1992 and Karatzas and Shreve, 1998 for the case of time-additive expected utility, and Schroder and Skiadas, 2003 and Appendix A of Schroder and Skiadas, 2005b for the case of recursive utility).

For expositional simplicity, in the remainder of this section we further assume that the relative risk aversion process $Q$ assumes the block diagonal 
structure

$$
Q=\left[\begin{array}{cc}
Q_{M M} & 0 \\
0 & Q_{N N}
\end{array}\right],
$$

where $Q_{M M} \in \mathcal{L}\left(\mathbb{R}^{m \times m}\right)$ and $Q_{N N} \in \mathcal{L}\left(\mathbb{R}^{(d-m) \times(d-m)}\right)$. In this context, the function $\mathcal{Q}$ of BSDE (42) of the optimality conditions can be written as

$$
\begin{aligned}
\mathcal{Q}\left(\sigma^{\lambda}\right)= & \sigma_{N}^{\lambda \prime} Q_{N N} \sigma_{N}^{\lambda}+2\left(\eta_{M}+\sigma_{M}^{\lambda}\right)^{\prime} \sigma_{M}^{\lambda} \\
& -\left(\eta_{M}+\sigma_{M}^{\lambda}\right)^{\prime} Q_{M M}^{-1}\left(\eta_{M}+\sigma_{M}^{\lambda}\right) .
\end{aligned}
$$

The corresponding optimal trading strategy is

$$
\psi_{M}=\left(Q_{M M} \sigma_{M}^{R}\right)^{-1}\left(\eta_{M}-\left(Q_{M M}-I_{M M}\right) \sigma_{M}^{\lambda}\right),
$$

where $I_{M M}$ is the $m \times m$ identity matrix.

Example 33 (Mean-variance efficiency). If $Q_{M M}=I_{M M}$, then $\psi_{M}$ is instantaneously mean-variance efficient, an observation that extends Example 31.

Example 34 (Fictitious market completion and duality). Consider the above incomplete-market setting, with the normalized return dynamics (44), where $m<d$, and the block-diagonal $Q$ in (46). Suppose that $\left(\lambda, \sigma^{\lambda}\right)$ solves the BSDE of the optimality conditions, $\left(\rho, \psi_{M}\right)$ is the corresponding optimal strategy, and $U$ is the corresponding optimal utility process. Given any choice of a nonmarketed-price-of-risk process $\eta_{N}$, we consider the complete market obtained by introducing $d-m$ fictitious assets, whose cumulative excess return process $R_{N}$ follows the dynamics $\mathrm{d} R_{N}=\eta_{N} \mathrm{~d} t+\mathrm{d} B_{N}$. The unique marketprice-of-risk process in this fictitious complete market is given by (45). We let $U^{\eta_{N}}$ denote the corresponding complete-market optimal utility process. Simple algebra shows that if one makes the specific selection

$$
\eta_{N}=\left(Q_{N N}-I_{N N}\right) \sigma_{N}^{\lambda},
$$

then $\left(\lambda, \sigma^{\lambda}\right)$ satisfies the BSDE of the optimality conditions in the fictitious complete market defined by this choice of $\eta_{N}$. Moreover, the corresponding optimal strategy in the fictitious complete market is $(\rho, \psi)$, where $\left(\rho, \psi_{M}\right)$ is the incomplete-market optimal strategy and $\psi_{N}=0$. In other words, the above specification of $\eta_{N}$ prices nonmarketed risk so that the investor finds it optimal to not trade the fictitious assets at all. As a consequence $U=U^{\eta_{N}}$. For any other choice of $\eta_{N},(\rho, \psi)$ need not be optimal in the fictitious complete market defined by $\eta_{N}$, and therefore $U \leqslant U^{\eta_{N}}$.

A different type of connection between incomplete and complete market solutions is given in the following example (which is generalized in Schroder and Skiadas, 2003). A particular case of the example shows that if the investor has the time-additive expected power utility (36) with $\gamma \in(0,2)$, then the 
solution to the investor's problem in an incomplete market is equivalent (in a sense clarified below) to the solution of the complete market problem obtained by pricing nontraded uncertainty risk-neutrally, and setting the investor's relative risk aversion toward nonmarketed uncertainty to $1 /(2-\gamma)$. The original additive-utility problem with incomplete markets is therefore equivalent to a complete-markets problem with recursive utility.

Example 35 (Market-incompletess and source-dependent risk aversion). We further specialize the quasiquadratic form (41) of the proportional aggregator by assuming that

$$
Q=\gamma I, \quad \text { where } \gamma \in(0,2) .
$$

In Example 26 we saw that this class includes cases of Epstein-Zin utility, as well as time-additive expected discounted power utility. Let $\left(\rho, \psi_{M}\right)$ be an incomplete-market optimal strategy, with corresponding shadow-price-ofwealth process $\lambda$, wealth process $W$, and utility process $U$.

We complete the market by introducing fictitious assets that are priced riskneutrally; that is, the price-of-nonmarketed risk process is zero $\left(\eta_{N}=0\right)$. We let the corresponding excess return dynamics be given by $R_{N}=B_{N}$. In the resulting fictitious complete market, we consider the optimal strategy, not of the original investor, but rather of a fictitious investor whose proportional aggregator is

$$
\bar{G}(t, c, \sigma)=g(t, c)-\frac{1}{2}\left(\gamma \sigma_{M}^{\prime} \sigma_{M}+\frac{1}{2-\gamma} \sigma_{N}^{\prime} \sigma_{N}\right) .
$$

In other words, the fictitious investor's relative risk aversion toward nonmarketed risk is modified from $\gamma$ to $1 /(2-\gamma)$. Let $(\bar{\rho}, \bar{\psi})$ be the optimal strategy of the fictitious investor in the fictitious complete market, and let $\bar{\lambda}, \bar{W}$ and $\bar{U}$ be the corresponding shadow-price-of wealth, wealth, and utility processes. Comparing optimality conditions, we observe that

$$
\begin{aligned}
& \bar{\lambda}=\lambda, \quad \bar{\rho}=\rho, \quad \bar{\psi}_{M}=\psi_{M}, \\
& \text { and } \quad \frac{\bar{W}_{t}}{W_{t}}=\frac{\bar{U}_{t}}{U_{t}}=\exp \left(\int_{0}^{t} \bar{\psi}_{N}^{\prime} \mathrm{d} B_{N}\right) .
\end{aligned}
$$

The incomplete market solution can therefore be immediately recovered from the fictitious complete-market solution. This is true even though the specification of the fictitious-investor preferences does not depend on market prices!

\subsection{Solutions based on quadratic BSDEs}

In this subsection, we discuss scale-invariant formulations in which the BSDE satisfied by $\log (\lambda)$ takes a quadratic form. For certain specifications of the return dynamics, the quadratic BSDE solution can be expressed as a 
quadratic function ${ }^{16}$ of an exogenous state process, with deterministic coefficients that solve an ODE system. This type of solution is familiar in Finance mainly in the context of risk-neutral pricing (see, for example, Duffie, 2005 and Piazzesi, 2005), where the relevant BSDE is linear. Our application extends the solution method to quadratic BSDEs, where the quadratic term reflects risk aversion. For expositional simplicity, we outline below only some examples, referring to Schroder and Skiadas (2003, 2005a, 2005b) for more general treatments. Examples of this type of solution can also be found in Chacko and Viceira (2005), Kim and Omberg (1996), Liu (2005), Schroder and Skiadas (1999), and Wachter (2002).

Continuing with the assumption of a homothetic recursive utility, we adopt the utility specification in the intersection of Examples 25 and 26; that is, the proportional aggregator is of the form

$$
G(t, x, \sigma)=\alpha+\beta \log (x)-\frac{\gamma}{2} \sigma^{\prime} \sigma,
$$

for some constants $\alpha \in \mathbb{R}$ and $\beta, \gamma \in \mathbb{R}_{++}$. The parameters $(\alpha, \beta)$ determine preferences over deterministic choices. Given $(\alpha, \beta)$, the parameter $\gamma$ adjusts risk aversion.

Remark 36. The treatment in Schroder and Skiadas (2003) allows possible source-dependent risk aversion, and parameters $\alpha$ and $\beta$ that are processes, the latter deterministic. The above specification for $\beta=0$ results in a utility that is ordinally equivalent to expected power utility for terminal consumption. Even though we have not covered the case of no intermediate consumption, essentially the same analysis applies. is

Recalling Example 25, the optimal strategy for the above utility specification

$$
\rho=\beta \quad \text { and } \quad \psi=\frac{1}{\gamma}\left(\sigma^{R \prime} \sigma^{R}\right)^{-1}\left(\mu^{R}-(\gamma-1) \sigma^{R \prime} \sigma^{\lambda}\right) .
$$

A myopic solution results for $\gamma=1$, corresponding to time-additive logarithmic utility (the intersection of Examples 25 and 31). The solution also simplifies if the investment opportunity set is deterministic, in which case $\sigma^{\lambda}=0$ (Example 30). To compute the optimal strategy with a stochastic investment opportunity set and $\gamma \neq 1$, we need to determine $\left(\lambda, \sigma^{\lambda}\right)$ by solving BSDE (42). Making the convenient change of variables

$$
\ell_{t}=\log \left(\lambda_{t}\right),
$$

\footnotetext{
${ }^{16}$ As explained in Schroder and Skiadas (2005b), the quadratic dependence on the state can be made affine by a suitable redefinition of the state process. A similar construction in a term-structure context appears in Cheng and Scaillet (2005).
} 
we note that $g^{*}(t, \lambda)=\alpha-\beta+\beta \log (\beta)-\beta \ell_{t}$. Direct computation shows that $\operatorname{BSDE}(42)$ can be written as a quadratic BSDE to be solved for $\ell$ :

$$
\mathrm{d} \ell_{t}=-\left(p_{t}-\beta \ell_{t}+h_{t}^{\prime} \sigma_{t}^{\ell}+\frac{1}{2} \sigma_{t}^{\ell \prime} H_{t} \sigma_{t}^{\ell}\right) \mathrm{d} t+\sigma_{t}^{\ell \prime} \mathrm{d} B, \quad \ell_{T}=0,
$$

where

$$
\begin{aligned}
& p=r+\alpha-\beta+\beta \log (\beta)+\frac{1}{2 \gamma} \mu^{R \prime}\left(\sigma^{R \prime} \sigma^{R}\right)^{-1} \mu^{R}, \\
& h=\frac{1-\gamma}{\gamma} \sigma^{R}\left(\sigma^{R \prime} \sigma^{R}\right)^{-1} \mu^{R}, \\
& H=(1-\gamma)\left[I+\frac{1-\gamma}{\gamma} \sigma^{R}\left(\sigma_{t}^{R \prime} \sigma^{R}\right)^{-1} \sigma^{R \prime}\right] .
\end{aligned}
$$

A general set of conditions under which the above quadratic BSDE can be reduced to an ODE is given in Schroder and Skiadas (2003). We only consider here two representative examples. As in the last subsection, we assume the normalization $\mathrm{d} R=\mu^{R} \mathrm{~d} t+\sigma_{M}^{R \prime} \mathrm{d} B_{M}$, and therefore the price-of-marketedrisk process is $\eta_{M}=\left(\sigma_{M}^{R \prime}\right)^{-1} \mu^{R}$. We outline the form of the solution below, leaving the details as an exercise.

Example 37. Given is an underlying $n$-dimensional state vector $Z$ following the dynamics

$$
\mathrm{d} Z=(\mu-\theta Z) \mathrm{d} t+\Sigma^{\prime} \mathrm{d} B,
$$

for some $\mu \in \mathbb{R}^{n}, \Sigma \in \mathbb{R}^{d \times n}$, and $\theta \in \mathbb{R}^{n \times n}$. The short-rate process and priceof-marketed-risk process are assumed to be given by

$$
r=C_{0}^{r}+C_{1}^{r \prime} Z+\frac{1}{2} Z^{\prime} C_{2}^{r} Z \text { and } \eta_{M}=C_{0}^{\eta}+C_{1}^{\eta \prime} Z,
$$

where the coefficients $C_{i}^{r}$ and $C_{i}^{\eta}$ are all constants of conforming dimensions. In this case, we conjecture a solution to BSDE (50) of the form

$$
\ell_{t}=C_{0}(t)+C_{1}(t)^{\prime} Z_{t}+\frac{1}{2} Z_{t}^{\prime} C_{2}(t) Z_{t},
$$

where the $C_{i}(t)$ are deterministic differentiable processes. Applying Itô's lemma to the above conjectured expression, collecting terms and comparing to the corresponding coefficients of BSDE (50) confirms that such a solution indeed solves BSDE (50), provided the coefficients $C_{i}$ solve an ODE system.

Example 38. We modify the above example by assuming the dynamics

$$
\begin{aligned}
& \mathrm{d} Z=(\mu-\theta Z) \mathrm{d} t+\Sigma^{\prime} \operatorname{diag}(\sqrt{v+V Z}) \mathrm{d} B, \\
& r=C_{0}^{r}+C_{1}^{r \prime} Z \quad \text { and } \quad \eta_{M}=\operatorname{diag}\left(\sqrt{v_{M}+V_{M} Z_{t}}\right) \varphi,
\end{aligned}
$$


where $\operatorname{diag}(x)$ denotes the diagonal matrix with $x$ on the diagonal, $\sqrt{x}$ denotes the vector with $i$ th element $\sqrt{x_{i}}$, and $\mu \in \mathbb{R}^{n}, \Sigma \in \mathbb{R}^{d \times n}, \theta \in \mathbb{R}^{n \times n}, C_{0}^{r} \in \mathbb{R}$, $C_{1}^{r} \in \mathbb{R}^{n}, v=\left[v_{M}^{\prime}, v_{N}^{\prime}\right]^{\prime} \in \mathbb{R}^{d}, V=\left[V_{M}^{\prime}, V_{N}^{\prime}\right]^{\prime} \in \mathbb{R}^{d \times n}, \varphi \in \mathbb{R}^{m}$. In this case, the conjectured solution takes the form

$$
\ell_{t}=C_{0}(t)+C_{1}(t)^{\prime} Z
$$

where again the $C_{i}(t)$ are differentiable deterministic processes. Arguing as in the last example, one obtains an ODE solved by $C_{1}$ alone, and another ODE (which uses the solution of the first ODE) that is satisfied by $C_{0}$. Given the pair $\left(C_{0}, C_{1}\right)$ satisfying the ODE pair, the above affine expression defines a solution to $\operatorname{BSDE}(50)$.

\subsection{Solutions with first-order risk aversion}

The final set of scale-invariant solutions we consider utilizes the kinked proportional aggregator of Section 4.4, representing source-dependent first-order risk aversion. More specifically, we assume the following condition, using the notation

$$
|x|^{\prime}=\left(\left|x_{1}\right|, \ldots,\left|x_{d}\right|\right), \quad x \in \mathbb{R}^{d} .
$$

Condition 39 (Quasi-quadratic proportional aggregator). Condition 28 holds with

$$
\mathcal{R}(\omega, t, \sigma)=\kappa(\omega, t)^{\prime}|\sigma|+\frac{1}{2} \sigma^{\prime} Q(\omega, t) \sigma,
$$

for some bounded processes $\kappa: \Omega \times[0, T] \rightarrow \mathbb{R}^{d}$ and $Q: \Omega \times[0, T] \rightarrow \mathbb{R}^{d \times d}$, where $Q(\omega, t)$ is diagonal and positive definite for all $(\omega, t)$.

We adopt the notation and return normalization of Section 5.2. In particular, the excess return dynamics and the marketed-price-of-risk process are

$$
\mathrm{d} R=\mu^{R} \mathrm{~d} t+\sigma_{M}^{R \prime} \mathrm{d} B_{M} \quad \text { and } \quad \eta_{M}=\left(\sigma_{M}^{R \prime}\right)^{-1} \mu_{M}^{R} .
$$

To formulate optimality conditions in this setting, we define, for any $\kappa \in \mathbb{R}_{+}$, the collar function

$$
C(\alpha ; \kappa)=\min \{\max \{0, \alpha-\kappa\}, \alpha+\kappa\}, \quad \alpha \in \mathbb{R},
$$


plotted below for $\kappa=1$ :

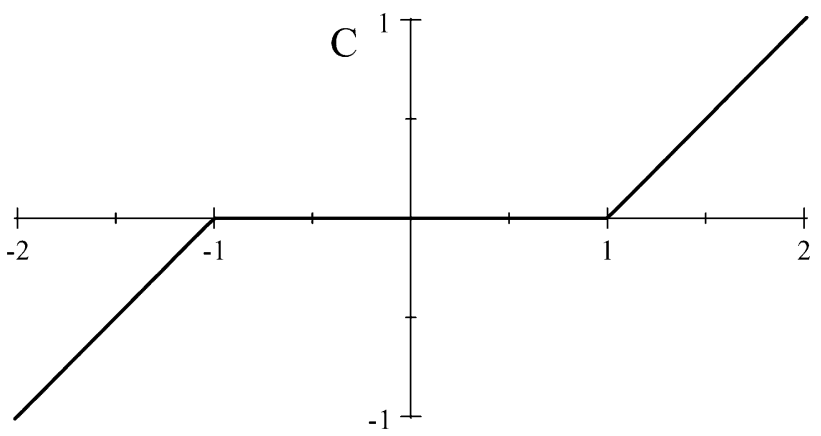

The collar function will be applied to vectors coordinate by coordinate:

$$
\begin{aligned}
& C(\alpha ; \kappa)=\left(C\left(\alpha_{1} ; \kappa_{1}\right), \ldots, C\left(\alpha_{m} ; \kappa_{m}\right)\right)^{\prime}, \\
& \quad \text { for any } \alpha \in \mathbb{R}^{m} \text { and } \kappa \in \mathbb{R}_{+}^{m} .
\end{aligned}
$$

The BSDE for $\lambda$ in this case is of the same form as in the smooth-quasiquadratic case, except that $\mathcal{Q}$ is replaced with the function $\mathcal{K}: \Omega \times[0, T] \times$ $\mathbb{R}^{d} \rightarrow \mathbb{R}$ defined by

$$
\begin{aligned}
\mathcal{K}(z)= & 2 \kappa_{N}^{\prime}\left|z_{N}\right|+z_{N}^{\prime} Q_{N N} z_{N}+2\left(\eta_{M}+z_{M}\right)^{\prime} z_{M} \\
& -C\left(\eta_{M}+z_{M} ; \kappa_{M}\right)^{\prime} Q_{M M}^{-1} C\left(\eta_{M}+z_{M} ; \kappa_{M}\right) .
\end{aligned}
$$

Expression (47) for $\mathcal{Q}$ is recovered if one sets $\kappa=0$.

With the above notation and return normalization in place, the optimality conditions (Condition 22) under Condition 39 reduce to the following steps:

1. The shadow-price-of-wealth process $\lambda$ solves the BSDE:

$$
\frac{\mathrm{d} \lambda_{t}}{\lambda_{t}}=-\left(r_{t}+g^{*}\left(t, \lambda_{t}\right)-\frac{1}{2} \mathcal{K}\left(t, \sigma_{t}^{\lambda}\right)\right) \mathrm{d} t+\sigma_{t}^{\lambda \prime} \mathrm{d} B_{t}, \quad \lambda_{T}=1 .
$$

2. Given the solution $\left(\lambda, \sigma^{\lambda}\right)$ from step one, the optimal strategy is

$$
\rho=\lambda \mathcal{I}^{g}(\lambda) \text { and } \psi=\left(\sigma_{M}^{R}\right)^{-1}\left[Q_{M M}^{-1} C\left(\eta_{M}+\sigma_{M}^{\lambda} ; \kappa_{M}\right)-\sigma_{M}^{\lambda}\right] .
$$

3. The wealth process generated by the strategy $(\rho, \psi)$ is computed from the budget equation, the corresponding optimal consumption plan is $c=$ $\rho W$, and its utility process is $U=\lambda W$.

The proof of the above claim (given in Schroder and Skiadas, 2003) is a matter of direct calculation using the specific aggregator form, and the key homogeneity condition at the optimum: $U=\lambda W$. The latter also implies that if $\mathrm{d} U / U=\ldots \mathrm{d} t+\sigma^{U^{\prime}} \mathrm{d} B$, then

$$
\sigma_{M}^{U}=\left(Q_{M M}\right)^{-1} C\left(\eta_{M}+\sigma_{M}^{\lambda} ; \kappa_{M}\right) .
$$


Consequently, for any $i \in M, \sigma^{U i}$ vanishes whenever $\eta_{M}^{i}+\sigma_{M}^{\lambda i} \in\left[-\kappa_{i}, \kappa_{i}\right]$. Such perfect hedging of utility risk with respect to some source of risk is not encountered with an aggregator derived from a smooth certainty equivalent. The following example (from Schroder and Skiadas, 2003) extends Section 5.3 of Chen and Epstein (2002). Further examples of nonparticipation as an expression of source-dependent first-order risk aversion can be found in Epstein and Miao (2003) and Schroder and Skiadas (2003).

Example 40 (Deterministic investment opportunity set). Suppose that $r, \mu^{R}$, $\sigma^{R}, \kappa$, and $Q$ are all deterministic. Then the solution simplifies by setting $\sigma^{\lambda}=0$. In particular, the optimal trading strategy is $\psi=\left(Q_{M M} \sigma_{M}^{R}\right)^{-1} \times$ $C\left(\eta_{M} ; \kappa_{M}\right)$. Let us further assume, for simplicity, that $\sigma_{M M}^{R}$ is diagonal with positive diagonal. For any $i \in M, \psi_{i}=0$ when $\eta_{i} \in\left[-\kappa_{i},+\kappa_{i}\right]$; the agent will not participate in the market for risk $i$, unless its instantaneous expected return relative to its risk is sufficiently far from zero. This type of solution can be combined with different belief specifications, as in Remark 15, to obtain a richer set of optimal portfolio holdings. In particular, adding the term $b^{\prime} \sigma$ to the proportional aggregator, for some (bounded) process $b$, means that the investor believes the market price of risk process to be $\eta-b$, rather than $\eta$, and therefore the investor will not participate in the market for risk source $i$ if $\eta_{i} \in\left[b_{i}-\kappa_{i}, b_{i}+\kappa_{i}\right]$. If we further assume that $b_{i}=-\kappa_{i}$, then the optimal holding of asset $i$ is $\psi_{i}=Q_{i i}^{-1} \mu_{i}^{R} /\left(\sigma_{i i}^{R}\right)^{2}$ when $\mu_{i}^{R}>0$ (just as with $\kappa=b=0$ ), but the agent will only short asset $i$ when $\mu_{i}^{R}<-2 \kappa_{i} \sigma_{i i}^{R}$. In other words, in this case, the optimal portfolio is identical to the Merton solution for positive expected excess returns, yet it is optimal for the investor to not go short for sufficiently small negative expected returns.

\section{Extensions}

This section concludes with two direct extensions of the main chapter's material, and a list of further topics and related references.

\subsection{Convex trading constraints}

We outline an extension of this chapter's arguments to include convex trading constraints, referring to Schroder and Skiadas (2003) for details. Examples of analysis of the Merton problem with constraints based on the HamiltonJacobi-Bellman approach include Zariphopoulou (1994) and Vila and Zariphopoulou (1997). Convex duality with trading constraints and additive utility is studied by He and Pearson (1991), Karatzas et al. (1991) (incomplete markets); Shreve and Xu (1992a, 1992b) (short-sale constraints); and Cvitanić and Karatzas (1992) (convex constraints). Related discussions with recursive preferences can be found in El Karoui et al. (2001), and Schroder and Skiadas 
(2003, 2005b). We will not discuss duality here. Also not discussed here are constraints that prevent the investor from borrowing against future income, which is the focus of He and Pagès (1993), El Karoui and Jeanblanc-Picquè (1998), and Detemple and Serrat (2003).

We consider this chapter's setting with the additional constraint that the investor's trading strategy must be valued in some given convex set $K \subseteq \mathbb{R}^{m}$ at all times. For example, $K=\mathbb{R}_{+}^{m}$ represents the impossibility of short-selling. The definition of a feasible cash flow now includes the requirement that it can be financed by a $K$-valued trading strategy. We let $\delta_{K}\left(\varepsilon_{t}\right)=\sup \left\{k^{\prime} \varepsilon_{t}: k \in K\right\}$ denote the support function of $K$.

We fix a feasible strategy $(\rho, \psi)$ financing the consumption plan $c$. Given our new notion of feasibility, the definition of a state-price density at $c$ is the same as before. The smaller the set $K$, the smaller the set of feasible incremental cash flows, and therefore the larger the set of state price densities at $c$. Under some regularity, state price dynamics are characterized in Schroder and Skiadas (2003) as

$$
\begin{gathered}
\frac{\mathrm{d} \pi_{t}}{\pi_{t}}=-\left(r_{t}+\delta_{K}\left(\varepsilon_{t}\right)\right) \mathrm{d} t-\eta_{t}^{\prime} \mathrm{d} B_{t}, \\
\varepsilon_{t}=\mu_{t}^{R}-\sigma_{t}^{R \prime} \eta_{t}, \quad \psi_{t}^{\prime} \varepsilon_{t}=\delta_{K}\left(\varepsilon_{t}\right) .
\end{gathered}
$$

Proposition 3 still applies here, so combining the above dynamics with those of a utility supergradient density results in sufficient optimality conditions as a constrained FBSDE system.

As in the unconstrained case, scale invariance results in the uncoupling of the forward and backward components of the BSDE system. For example, consider a scale-invariant recursive utility with the smooth quasi-quadratic proportional aggregator of Condition 29, a specification that includes expected discounted power utility and Epstein-Zin utility (see Example 26). As shown in Schroder and Skiadas (2003), in this case the optimality conditions can be written as the constrained BSDE:

$$
\begin{aligned}
& \frac{\mathrm{d} \lambda_{t}}{\lambda_{t}}=-\left(r_{t}+\delta_{K}\left(\varepsilon_{t}\right)+g^{*}\left(t, \lambda_{t}\right)-\frac{1}{2} \sigma_{t}^{\lambda \prime} Q_{t} \sigma_{t}^{\lambda}\right. \\
&\left.+\frac{1}{2} \psi_{t}^{\prime} \sigma_{t}^{R \prime} Q_{t} \sigma_{t}^{R} \psi_{t}\right) \mathrm{d} t+\sigma_{t}^{\lambda \prime} \mathrm{d} B_{t}, \quad \lambda_{T}=1, \\
& \psi_{t}=\left(\sigma_{t}^{R \prime} Q_{t} \sigma_{t}^{R}\right)^{-1}\left(\mu_{t}^{R}-\varepsilon_{t}-\sigma_{t}^{R^{\prime}}\left(Q_{t}-I\right) \sigma_{t}^{\lambda}\right) \in K, \\
& \psi_{t}^{\prime} \varepsilon_{t}=\delta_{K}\left(\varepsilon_{t}\right) .
\end{aligned}
$$

Example 41. Under Condition 29, a particularly simple expression for the optimal trading strategy is obtained if $K=\left\{k \in \mathbb{R}^{m}: \alpha \leqslant l^{\prime} k \leqslant \beta\right\}$ where $l \in \mathbb{R}^{m}$ and $\alpha$ and $\beta$ are valued in $[-\infty,+\infty]$. The case of a short-sale constraint on asset $i$ corresponds to $\alpha=0, \beta=\infty$, and $l$ a vector of zeros except for a one in the $i$ th position. The case of a cap on the proportion of wealth borrowed, possibly combined with a limit on short sales as a fraction of wealth, corresponds 
to letting $l$ be a vector of ones. We assume that $K$ is nonempty, and define

$$
\psi_{t}^{*}=A_{t}\left(\mu_{t}^{R}-\sigma_{t}^{R^{\prime}}\left(Q_{t}-I\right) \sigma_{t}^{\lambda}\right), \quad A_{t}=\left(\sigma_{t}^{R \prime} Q_{t} \sigma_{t}^{R}\right)^{-1} .
$$

The above expression gives the optimal trading strategy as a function of $\sigma^{\lambda}$ in the unconstrained case $(\alpha=-\infty, \beta=\infty)$. The (constrained) optimal trading strategy $\psi$ and process $\varepsilon$ in the dynamics of $\lambda$ are given by

$$
\psi_{t}=\psi_{t}^{*}-A_{t} \varepsilon_{t}, \quad \varepsilon_{t}=-\left(l^{\prime} A_{t} l\right)^{-1}\left[\min \left\{\max \left\{l^{\prime} \psi_{t}^{*}, \alpha\right\}, \beta\right\}-l^{\prime} \psi_{t}^{*}\right] l .
$$

These equations can in turn be used to complete the specification of the BSDE for $\lambda$, which can then be solved by some numerical method (for example, with numerical PDE methods in a Markovian setting).

\subsection{Translation-invariant formulations and nontradeable income}

A parallel theory to this chapter's scale-invariance argument is based on a notion of translation invariance in a setting that allows for a nontradeable income stream. This type of formulation is familiar in the subclass of problems with expected discounted exponential utility and Gaussian dynamics, as, for example, in Svensson and Werner (1993) and Musiela and Zariphopoulou (2004). We outline below a formulation with recursive utility, which is a special case of Schroder and Skiadas (2005a, 2005b) (where trading constraints, nonlinear wealth dynamics, and unpredictable return jumps are also considered).

We modify our earlier setting by assuming that the investor is endowed with a possibly nontradeable cash flow $e$, in addition to the initial wealth $w_{0}$. Consumption in this subsection is allowed to take negative values, and financial wealth can vanish. The representation of portfolios in terms of proportions of wealth is therefore unsuitable in our new setting. We correct this by defining a trading plan to be a process $\phi \in \mathcal{L}\left(\mathbb{R}^{m}\right)$, where $\phi_{t}^{i}$ represents a dollar amount invested in asset $i$ at time $t$. The dollar amount invested in the money market at time $t$ is $W_{t}-\sum_{i=1}^{m} \phi_{t}^{i}$, where $W_{t}$ represents total time- $t$ financial wealth (excluding $e$ ). Ignoring some integrability requirements, a plan is a triple $(c, \phi, W)$ of a consumption plan $c$, a trading plan $\phi$, and a wealth process $W$. The plan $(c, \phi, W)$ is feasible if it satisfies the budget equation:

$$
W_{0}=w, \quad \mathrm{~d} W_{t}=\left(r_{t} W_{t}+e_{t}-c_{t}\right) \mathrm{d} t+\phi_{t}^{\prime} \mathrm{d} R_{t}, \quad c_{T}=W_{T}+e_{T} .
$$

The derivation and form of the optimality conditions as a FBSDE system in this setting is similar to this chapter's main analysis, as explained in Schroder and Skiadas (2005a, 2005b).

We place restrictions on the market and preferences in terms of a strictly positive (bounded) cash flow $\gamma$, that is fixed throughout. On the market side, we assume there is a tradeable fund that generates $\gamma$ as a dividend stream. We refer to this fund as the " $\gamma$-fund," and we let $\Gamma_{t}$ and $\kappa_{t}$ be its time- $t$ value and 
value allocation, respectively. ${ }^{17}$ The $\gamma$-fund budget equation is

$$
\mathrm{d} \Gamma_{t}=\left(r_{t} \Gamma_{t}-\gamma_{t}\right) \mathrm{d} t+\Gamma_{t} \kappa_{t}^{\prime} \mathrm{d} R_{t}, \quad \Gamma_{T}=\gamma_{T} .
$$

For example, if $r$ and $\gamma$ are deterministic, the $\gamma$-fund can be implemented entirely through the money market (with $\kappa=0$ ). If either $r$ or $\gamma$ is stochastic, one can assume that risky asset one is a share in the $\gamma$-fund, and therefore $\kappa=(1,0, \ldots, 0)$.

On the preference side, we assume that the investor's time-zero utility function is translation-invariant with respect to $\gamma$, meaning that, for any consumption plans $a$ and $b$,

$$
U_{0}(a)=U_{0}(b) \quad \text { implies } \quad U_{0}(a+k \gamma)=U_{0}(b+k \gamma) \quad \text { for all } k \in \mathbb{R} .
$$

If utility is normalized so that the investor is indifferent between the consumption plan $c$ and the consumption plan $U_{0}(c) \gamma$, the above property can equivalently be stated as quasilinearity with respect to $\gamma$; that is, $U_{0}(c+k \gamma)=$ $U_{0}(c)+k$ for any consumption plan $c$ and scalar $k$. For recursive utility, the latter restriction is essentially equivalent to the BSDE form:

$$
\mathrm{d} U_{t}=-G\left(t, \frac{c_{t}}{\gamma_{t}}-U_{t}, \Sigma_{t}\right) \mathrm{d} t+\Sigma_{t}^{\prime} \mathrm{d} B_{t}, \quad U_{T}=\frac{c_{T}}{\gamma_{T}},
$$

for a possibly state-dependent function $G$ that we call an absolute aggregator. For concreteness, we combine the above representation with our earlier formulation of possibly source-dependent risk aversion with a smooth conditional certainty equivalent, resulting in the quasi-quadratic absolute aggregator specification

$$
G(t, x, \Sigma)=g(t, x)-\frac{1}{2} \Sigma^{\prime} Q_{t} \Sigma
$$

In the remainder of this subsection, we assume this absolute aggregator form, where $g(t, x)$ is strictly increasing, concave, and differentiable in $x$, the partial derivative $g_{x}(t, \cdot)$ maps $\mathbb{R}$ onto $\mathbb{R}$, and $Q$ is a (bounded) process valued in the space of positive-definite $d \times d$ matrices.

Example 42 (Expected discounted exponential utility). Let $\beta$ be any (say bounded) process, and suppose the utility process $V$ of the plan $c$ is well defined by

$$
V_{t}=E_{t}\left[\int_{t}^{T}-\exp \left(-\int_{t}^{s} \beta_{u} \mathrm{~d} u-\frac{c_{s}}{\gamma_{s}}\right) \mathrm{d} s-\exp \left(-\int_{t}^{T} \beta_{u} \mathrm{~d} u-\frac{c_{T}}{\gamma_{T}}\right)\right] .
$$

\footnotetext{
${ }^{17}$ In Schroder and Skiadas (2005a) $\varrho=\Gamma \kappa$ was set, without loss in generality, equal to a constant for simplicity of exposition. Their analysis applies essentially unchanged with $\varrho$ stochastic, as assumed here and in Schroder and Skiadas (2005b) (where the exposition is simplified by taking $\kappa$ to be constant).
} 
Then the ordinally equivalent utility process $U_{t}=-\log \left(-V_{t}\right)$ solves BSDE (53) with the absolute aggregator (54), where $Q(\omega, t)=1$ and $g(\omega, t, x)=$ $\beta(\omega, t)-\exp (-x)$.

Analogously to the scale-invariance argument, translation-invariance with respect to $\gamma$ uncouples the FBSDE of the first-order conditions. Intuitively, if the agent's problem is solved at some information spot at a given financial wealth level, it is also solved at all wealth levels, since the agent can always invest any additional wealth to the $\gamma$-fund while preserving optimality.

More specifically, at the optimum, the utility process $U$, the wealth process $W$, and the shadow-price-of-wealth process $\lambda$, are related by

$$
U_{t}=\frac{1}{\Gamma_{t}}\left(Y_{t}+W_{t}\right) \quad \text { and } \quad \lambda_{t}=\frac{1}{\Gamma_{t}},
$$

where the process $Y$ solves the quadratic BSDE

$$
\begin{aligned}
\mathrm{d} Y_{t} & =-\left(e_{t}+p_{t}-r_{t} Y_{t}+\Sigma_{t}^{Y \prime} h_{t}+\frac{1}{2} \Sigma_{t}^{Y \prime} H_{t} \Sigma_{t}^{Y}\right) \mathrm{d} t+\Sigma_{t}^{Y \prime} \mathrm{d} B_{t}, \\
Y_{T} & =e_{T},
\end{aligned}
$$

with

$$
\begin{aligned}
& p=\Gamma g^{*}\left(\frac{\gamma}{\Gamma}\right)+\frac{\Gamma}{2}\left(\mu^{R}-\sigma_{t}^{R \prime} \sigma_{t}^{R} \kappa\right)^{\prime}\left(\sigma^{R \prime} Q \sigma^{R}\right)^{-1}\left(\mu^{R}-\sigma_{t}^{R \prime} \sigma_{t}^{R} \kappa\right), \\
& h=-\sigma^{R} \kappa-Q \sigma^{R}\left(\sigma^{R \prime} Q \sigma^{R}\right)^{-1}\left(\mu^{R}-\sigma_{t}^{R \prime} \sigma_{t}^{R} \kappa\right), \\
& H=\frac{1}{\Gamma}\left(Q \sigma^{R}\left(\sigma^{R \prime} Q \sigma^{R}\right)^{-1} \sigma^{R^{\prime}} Q-Q\right) .
\end{aligned}
$$

The optimal plan trading plan $\phi$ and consumption plan $c$ can be written as

$$
\begin{aligned}
& \phi=\phi^{0}+U \Gamma \kappa, \quad c=\gamma U+\gamma g_{x}^{-1}\left(\frac{\gamma}{\Gamma}, \frac{\sigma^{R} \phi^{0}+\Sigma^{Y}}{\Gamma}\right), \\
& \text { where } \phi^{0}=\left(\sigma^{R \prime} Q \sigma^{R}\right)^{-1}\left[\Gamma\left(\mu^{R}-\sigma^{R \prime} \sigma^{R} \kappa\right)-\sigma^{R \prime} Q \Sigma^{Y}\right] .
\end{aligned}
$$

Just as with the quadratic BSDE case of the scale-invariant formulation, for a certain class of price dynamics the solution reduces to an ODE system. We refer to Schroder and Skiadas (2005a, 2005b) for examples and extensions (some of which are outlined below).

\subsection{Other directions}

We conclude with a list of selected topics on dynamic portfolio theory and a highly biased small sample of associated references that can be consulted for further leads to a large related literature. Brandt (forthcoming) reviews the econometrics of portfolio choice. 
Nonlinear wealth dynamics. Cuoco and Cvitanić (1998), El Karoui et al. (2001), and Schroder and Skiadas (2005b) characterize optimality with wealth dynamics that can allow nonlinearities reflecting, for example, market impact or differential borrowing and lending rates. The last reference includes the extension of this chapter's scale/translation invariance arguments to this case.

Discontinuous information. Merton's original work includes examples of discontinuous information generated by Poisson jumps. The extension of Merton's work to Lévy type processes using the Hamilton-Jacobi-Bellman approach is presented in the monograph by Øksendal and Sulem (2005). This chapter's arguments are extended in Schroder and Skiadas (2005b) so that the filtration is generated by Brownian motions as well as marked point processes. The above references provide links to several other papers on this topic.

Habit formation. Asset pricing models with habit formation include Sundaresan (1989), Constantinides (1990), and Detemple and Zapatero (1991). Duffie and Skiadas (1994) defined recursive utility with habit formation, and computed its gradient density. The latter can be combined with this chapter's state price dynamics to formulate optimality conditions as a FBSDE system. Schroder and Skiadas (2002) showed that, by redefining consumption, a formulation with linear habit formation can be transformed to an equivalent one without habit formation. This technique can be used to mechanically translate this chapter's solutions (assuming either a deterministic short-rate process or complete markets) to corresponding solutions that incorporate linear habit formation. The same argument applies with durability of consumption.

Nontradeable income. We have seen that the optimality conditions given a nontradeable income simplify in the translation-invariant formulation, which implies constant absolute risk aversion. More general models of nontradeable income must deal with a fully coupled FBSDE system. The Merton problem with nontradeable income and additive utility has been analyzed in terms of the Hamilton-Jacobi-Bellman approach by Duffie and Zariphopoulou (1993), Duffie et al. (1997), and Koo (1998). Related theoretical results with nontradeable income and additive utilities include Cuoco (1997), Kramkov and Schachermeyer (1999, 2003), Cvitanić et al. (2001), and Hugonnier and Kramkov (2002).

Endogenous labor supply and retirement. Bodie et al. (1992), Bodie et al. (2004), Dybvig and Liu (2005), Farhi and Panageas (2005), and Liu and Neis (2002), among others, have analyzed the lifetime consumption-portfolio problem with endogenous labor supply and/or retirement. Recursive utility formulations in this area are yet to be developed.

Transaction costs. The Merton analysis has been extended to include proportional transaction costs by Davis and Norman (1990), Shreve and Soner (1994), 
Liu and Loewenstein (2002), and others. Grossman and Laroque (1990) and Cuoco and Liu (2000) studied problems in which transaction costs apply to changes in the stock of a durable good. Proportional transaction costs preserve scale invariance, motivating the use of expected discounted power utility in the above papers. Fixed transaction costs on the other hand destroy scale invariance. For this reason existing analytically tractable formulations with fixed transaction costs are based on translation invariance, so far only with additive exponential utility, as in Vayanos (1998) and Liu (2004). Optimality conditions with both proportional and fixed transaction costs with i.i.d. returns are given in Øksendal and Sulem (2002). This is only a small sample of a large literature dealing with some form of transaction costs. I am not aware of any related theoretical results with recursive utility.

\section{Acknowledgements}

I am grateful to Mark Schroder for the many years of collaborative research on which this article is based, as well as corrections to this chapter. I also thank Darrell Duffie, Flavio de Andrade, Ali Lazrak, Hong Liu, Jacob Sagi, George Skoulakis, Jeremy Staum, and Jared Williams for valuable feedback. I am responsible for all errors. The latest version of this article can be found at http://www.kellogg.nwu.edu/faculty/skiadas/home.htm.

\section{References}

Anderson, E., Hansen, L., Sargent, T. (2000). Robustness, detection and the price of risk. Working paper, Department of Economics, University of Chicago.

Arrow, K.J. (1965). Aspects of the Theory of Risk Bearing. Yrjo Jahnssonin Saatio, Helsinki.

Arrow, K.J. (1970). Essays in the Theory of Risk Bearing. North-Holland, London.

Bally, V., Pages, G. (2002). A quantization algorithm for solving discrete time multidimensional optimal stopping problems. Bernoulli 9, 1003-1049.

Bodie, Z., Merton, R.C., Samuelson, W.F. (1992). Labor supply flexibility and portfolio choice in a life cycle model. Journal of Economic Dynamics and Control 16, 427-449.

Bodie, Z., Detemple, J.B., Otruba, S., Walter, S. (2004). Optimal consumption-portfolio choices and retirement planning. Journal of Economic Dynamics and Control 28, 1115-1148.

Bouchard, B., Elie, R. (2005). Discrete time approximation of decoupled forward-backward SDE with jumps. Working paper, LPMA, CNRS, UMR 7599, Université Paris 6 and CREST.

Bouchard, B., Touzi, N. (2004). Discrete-time approximation and Monte-Carlo simulation of backward stochastic differential equations. Stochastic Processes and their Applications 111, 175-206.

Brandt, M.W. (forthcoming). Portfolio choice problems. In: Ait-Sahalia, Y., Hansen, L.P. (Eds.), Handbook of Financial Econometrics, Elsevier/North-Holland, New York.

Campbell, J., Viceira, L. (2002). Strategic Asset Allocation. Oxford Univ. Press, New York.

Chacko, G., Viceira, L. (2005). Dynamic consumption and Portfolio Choice with Stochastic Volatility in Incomplete Markets. Review of Financial Studies 18, 1369-1402.

Chen, Z., Epstein, L. (2002). Ambiguity, risk, and asset returns in continuous time. Econometrica 70, 1403-1443.

Cheng, P., Scaillet, O. (2005). Linear-quadratic jump-diffusion modeling with application to stochastic volatility. Working paper, HEC Geneva, Switzerland. 
Chevance, D. (1997). Numerical methods for backward stochastic differential equations. In: Rogers, L., Talay, D. (Eds.), Numerical Methods in Finance. Cambridge Univ. Press, Cambridge, UK, pp. 232244.

Constantinides, G.M. (1990). Habit formation: A resolution of the equity premium puzzle. Journal of Political Economy 98, 519-543.

Cox, J., Huang, C.-F. (1989). Optimal consumption and portfolio policies when asset prices follow a diffusion process. Journal of Economic Theory 49, 33-83.

Cuoco, D. (1997). Optimal consumption and equilibrium prices with portfolio constraints and stochastic income. Journal of Economic Theory 72, 33-73.

Cuoco, D., Cvitanić, J. (1998). Optimal consumption choices for a large investor. Journal of Economic Dynamics and Control 22, 401-436.

Cuoco, D., Liu, H. (2000). Optimal consumption of a divisible durable good. Journal of Economic Dynamics and Control 24, 561-613.

Cvitanić, J., Karatzas, I. (1992). Convex duality in constrained portfolio optimization. The Annals of Applied Probability 2, 767-818.

Cvitanić, J., Schachermayer, W., Wang, H. (2001). Utility maximization in incomplete markets with random endowment. Finance and Stochastics 5, 259-272.

Daniel, K., Hirshleifer, D., Teoh, S.H. (2002). Investor psychology in capital markets: Evidence and policy implications. Journal of Monetary Economics 49, 139-209.

Davis, M., Norman, A.R. (1990). Portfolio selection with transaction costs. Mathematics of Operations Research 15, 676-713.

Detemple, J., Serrat, A. (2003). Dynamic equilibrium with liquidity constraints. Review of Financial Studies 16, 597-629.

Detemple, J., Zapatero, F. (1991). Asset prices in an exchange economy with habit formation. Econometrica 59, 1633-1657.

Douglas Jr., J., Ma, J., Protter, P. (1996). Numerical methods for forward-backward stochastic differential equations. Annals of Applied Probability 6, 940-968.

Duffie, D. (2001). Dynamic Asset Pricing Theory, third ed. Princeton Univ. Press, Princeton, New Jersey.

Duffie, D. (2005). Credit risk modeling with affine processes. Journal of Banking and Finance 29, 27512802.

Duffie, D., Epstein, L. (1992). Stochastic differential utility. Econometrica 60, 353-394.

Duffie, D., Skiadas, C. (1994). Continuous-time security pricing: A utility gradient approach. Journal of Mathematical Economics 23, 107-131.

Duffie, D., Zariphopoulou, T. (1993). Optimal investment with undiversifiable income risk. Mathematical Finance 3, 135-148.

Duffie, D., Fleming, V., Soner, M., Zariphopoulou, T. (1997). Hedging in incomplete markets with HARA utility. Journal of Economic Dynamics and Control 21, 753-782.

Dybvig, P.H., Liu, H. (2005). Lifetime consumption and investment: Retirement and constrained borrowing. Working paper, Olin School of Business, Washington University, St. Louis, MO.

El Karoui, N., Jeanblanc-Picquè, M. (1998). Optimization of consumption with labor income. Finance and Stochastics 2, 409-440.

El Karoui, N., Mazliak, L. (Eds.) (1997). Backward Stochastic Differential Equations. AddisonWesley/Longman, Essex, UK.

El Karoui, N., Peng, S., Quenez, M.-C. (1997). Backward stochastic differential equations in finance. Mathematical Finance 7, 1-71.

El Karoui, N., Peng, S., Quenez, M.-C. (2001). A dynamic maximum principle for the optimization of recursive utilities under constraints. Annals of Applied Probability 11, 664-693.

Ellsberg, D. (1961). Risk, ambiguity, and the savage axioms. Quarterly Journal of Economics 75, 643669.

Epstein, L. (1992). Behavior under risk: Recent developments in theory and applications. In: Laffont, J.-J. (Ed.), Advances in Economic Theory. Cambridge Univ. Press, Cambridge, UK.

Epstein, L., Miao, J. (2003). A two-person dynamic equilibrium under ambiguity. Journal of Economic Dynamics and Control 27, 1253-1288.

Epstein, L., Schneider, M. (2003). Recursive multiple priors. Journal of Economic Theory 113, 1-31. 
Epstein, L., Zin, S. (1989). Substitution, risk aversion, and the temporal behavior of consumption and asset returns: A theoretical framework. Econometrica 57, 937-969.

Farhi, E., Panageas, S. (2005). Saving and investing for early retirement: A theoretical analysis. Working paper, Department of Economics, MIT, Cambridge, MA.

Fleming, W.H., Soner, H.M. (1993). Controlled Markov Processes and Viscosity Solutions. Springer, New York.

Giovannini, A., Weil, P. (1989). Risk aversion and intertemporal substitution in the capital asset pricing model. NBER working paper No. 2824, Cambridge, MA.

Gobet, E., Lemor, J., Warin, X. (2005). A regression-based Monte Carlo method to solve backward stochastic differential equations. Annals of Applied Probability 15, 2172-2202.

Gollier, C. (2001). The Economics of Risk and Time. MIT Press, Cambridge, MA.

Grossman, S.J., Laroque, G. (1990). Asset pricing and optimal portfolio choice in the presence of illiquid durable consumption goods. Econometrica 58, 25-51.

Hamadene, S. (1996). Équations différentielles stochastiques rétrogrades : Le cas localement Lipschitzien. Annales de l'Institut Henri Poincaré 32, 645-659.

Hansen, L., Sargent, T., Turmuhambetova, G., Williams, N. (2001). Robustness and uncertainty aversion. Working paper, Department of Economics, University of Chicago.

He, H., Pagès, H. (1993). Labor income, borrowing constraints and equilibrium asset prices: A duality approach. Economic Theory 3, 663-696.

He, H., Pearson, N. (1991). Consumption and portfolio policies with incomplete markets and short-sale constrains: The infinite dimensional case. Journal of Economic Theory 54, 259-304.

Hugonnier, J., Kramkov, D. (2002). Optimal investment with random endowments in incomplete markets. Working paper, HEC Montreal and Carnegie Mellon University.

Karatzas, I., Shreve, S. (1988). Brownian Motion and Stochastic Calculus. Springer, New York.

Karatzas, I., Shreve, S. (1998). Methods of Mathematical Finance. Springer, New York.

Karatzas, I., Lehoczky, J., Shreve, S. (1987). Optimal portfolio and consumption decisions for a 'small investor' on a finite horizon. SIAM Journal of Control and Optimization 25, 1557-1586.

Karatzas, I., Lehoczky, J., Shreve, S., Xu, G. (1991). Martingale and duality methods for utility maximization in an incomplete market. SIAM Journal of Control and Optimization 29, 702-730.

Kim, T., Omberg, E. (1996). Dynamic nonmyopic portfolio behavior. Review of Financial Studies 9, $141-$ 162.

Klibanoff, P., Marinacci, M., Mukerji, S. (2002). A smooth model of decision making under ambiguity. Working paper, MEDS, Kellogg School of Management, Northwestern University.

Kobylanski, M. (2000). Backward stochastic differential equations and partial differential equations with quadratic growth. The Annals of Probability 28, 558-602.

Koo, H. (1998). Nontraded assets in incomplete markets. Mathematical Finance 8, 49-65.

Korn, R. (1997). Optimal Portfolios. World Scientific, River Edge, NJ.

Kramkov, D., Schachermayer, W. (1999). The asymptotic elasticity of utility functions and optimal investment in incomplete markets. Annals of Applied Probability 9, 904-950.

Kramkov, D., Schachermayer, W. (2003). Necessary and sufficient conditions in the problem of optimal investment in incomplete markets. Annals of Applied Probability 13, 1504-1516.

Kreps, D., Porteus, E. (1978). Temporal resolution of uncertainty and dynamic choice theory. Econometrica 46, 185-200.

Lazrak, A., Quenez, M.C. (2003). A generalized stochastic differential utility. Mathematics of Operations Research 28, 154-180.

Lemor, J., Gobet, E., Warin, X. (2006). Rate of convergence of an empirical regression method for solving generalized backward stochastic differential equations. Bernoulli 12, 889-916.

Lepeltier, J.-P., San Martín, J. (1997). Backward stochastic differential equations with continuous coefficient. Statistics and Probability Letters 32, 425-430.

Lepeltier, J.-P., San Martín, J. (1998). Existence for BSDE with superlinear-quadratic coefficient. Stochastics and Stochastics Reports 63, 227-240.

Lepeltier, J.-P., San Martín, J. (2002). On the existence or non-existence of solutions for certain backward stochastic differential equations. Bernoulli 8, 123-137. 
Liu, H. (2004). Optimal consumption and investment with transaction costs and multiple risky assets. Journal of Finance 59, 289-338.

Liu, J. (2005). Portfolio selection in stochastic environments. Working paper, UCLA.

Liu, H., Loewenstein, M. (2002). Optimal portfolio selection with transaction costs and finite horizons. Review of Financial Studies 15, 805-835.

Liu, J., Neis, E. (2002). Endogenous retirement and portfolio choice. Working paper, UCLA.

Ma, J., Yong, J. (1999). Forward-Backward Stochastic Differential Equations and Their Applications. Springer-Verlag, Berlin/Heidelberg.

Ma, J., Protter, P., Yong, J. (1994). Solving forward-backward stochastic differential equations explicitly-A four step scheme. Probability Theory and Related Fields 98, 339-359.

Ma, J., Protter, P., San Martin, J., Torres, S. (2002). Numerical method for backward stochastic differential equations. Annals of Applied Probability 12, 302-316.

Maenhout, P. (1999). Robust portfolio rules and asset pricing. Working paper, INSEAD.

Markowitz, H. (1952). Portfolio selection. Journal of Finance 7, 77-91.

Merton, R. (1969). Lifetime portfolio selection under uncertainty: The continuous time case. Review of Economics and Statistics 51, 247-257.

Merton, R. (1971). Optimum consumption and portfolio rules in a continuous-time model. Journal of Economic Theory 3, 373-413;

Erratum Journal of Economic Theory 6 (1973) 213-214.

Merton, R. (1990). Continuous Time Finance. Blackwell, Malden, MA.

Musiela, M., Zariphopoulou, T. (2004). An example of indifference prices under exponential preferences. Finance and Stochastics 8, 229-239.

Narens, L. (1985). Abstract Measurement Theory. MIT Press, Cambridge, MA.

Obstfeld, M. (1994). Risk-taking, global diversification, and growth. American Economic Review 84, 1310-1329.

$\varnothing$ ksendal, B., Sulem, A. (2002). Optimal consumption and portfolio with fixed and proportional transaction costs. SIAM Journal of Control and Optimization 40, 1765-1790.

$\varnothing \mathrm{ksendal}, \mathrm{B} .$, Sulem, A. (2005). Applied Stochastic Control of Jump Diffusions. Springer, New York.

Pardoux, E., Peng, S. (1990). Adapted solution of a backward stochastic differential equation. Systems and Control Letters 14, 55-61.

Piazzesi, M. (2005). Affine term structure models. Handbook of Financial Econometrics. Elsevier/NorthHolland, Amsterdam/New York.

Pratt, J.W. (1964). Risk aversion in the small and in the large. Econometrica 32, 122-136.

Schroder, M., Skiadas, C. (1999). Optimal consumption and portfolio selection with stochastic differential utility. Journal of Economic Theory 89, 68-126.

Schroder, M., Skiadas, C. (2002). An isomorphism between asset pricing models with and without linear habit formation. Review of Financial Studies 15, 1189-1221.

Schroder, M., Skiadas, C. (2003). Optimal lifetime consumption-portfolio strategies under trading constraints and generalized recursive preferences. Stochastic Processes and Their Applications 108, $155-202$.

Schroder, M., Skiadas, C. (2005a). Lifetime consumption-portfolio choice under trading constraints and nontradeable income. Stochastic Processes and Their Applications 115, 1-30.

Schroder, M., Skiadas, C. (2005b). Optimality and state pricing in constrained financial markets with recursive utility under continuous and discontinuous information. Mathematical Finance, in press.

Segal, U., Spivak, A. (1990). First-order versus second-order risk aversion. Journal of Economic Theory $51,111-125$.

Sethi, S. (1997). Optimal Consumption and Investment with Bankruptcy. Kluwer Academic Publishers, Norwell, MA.

Shreve, S., Soner, H.M. (1994). Optimal investment and consumption with transaction costs. Annals of Applied Probability 4, 609-692.

Shreve, S., Xu, G. (1992a). A duality method for optimal consumption and investment under shortselling prohibition. I. General market coefficients. Annals of Applied Probability 2, 87-112.

Shreve, S., Xu, G. (1992b). A duality method for optimal consumption and investment under shortselling prohibition. II. Constant market coefficients. Annals of Applied Probability 2, 314-328. 
Skiadas, C. (1998). Recursive utility and preferences for information. Economic Theory 12, 293-312.

Skiadas, C. (2003). Robust control and recursive utility. Finance and Stochastics 7, 475-489.

Sundaresan, S. (1989). Intertemporally dependent preferences and the volatility of consumption and wealth. Review of Financial Studies 2, 73-89.

Svensson, L. (1989). Portfolio choice with non-expected utility in continuous time. Economic Letters 30, 313-317.

Svensson, L., Werner, I. (1993). Nontraded assets in incomplete markets: Pricing and portfolio choice. European Economic Review 37, 1149-1168.

Uppal, R., Wang, T. (2003). Model misspecification and under-diversification. Journal of Finance.

Vayanos, D. (1998). Transaction costs and asset prices: A dynamic equilibrium model. Review of Financial Studies 11, 1-58.

Vila, J.-L., Zariphopoulou, T. (1997). Optimal consumption and portfolio choice with borrowing constraints. Journal of Economic Theory 77, 402-431.

von Neumann, J., Morgenstern, O. (1944). Theory of Games and Economic Behavior. Princeton Univ. Press, Princeton, NJ.

Wachter, J. (2002). Portfolio and consumption decisions under mean-reverting returns: An exact solution in complete markets. Journal of Financial and Quantitative Analysis 37, 63-91.

Wakker, P.P. (1989). Additive Representations of Preferences. Kluwer, Dordrecht.

Yong, J., Zhou, X.Y. (1999). Stochastic Controls: Hamiltonian Systems and HJB Equations. SpringerVerlag, New York.

Zariphopoulou, T. (1994). Consumption-investment models with constraints. SIAM Journal on Control and Optimization 32, 59-85.

Zariphopoulou, T., Tiu, C.-I. (2002). Optimization in incomplete markets with recursive utility. Working paper, University of Texas, Austin.

Zhang, J. (2004). A numerical scheme for BSDEs. Annals of Applied Probability 14, 459-488. 